Article

\title{
Small-RNA Sequencing Reveals Altered Skeletal Muscle microRNAs and snoRNAs Signatures in Weanling Male Offspring from Mouse Dams Fed a Low Protein Diet during Lactation
}

\author{
Ioannis Kanakis ${ }^{1,2, *}$, Moussira Alameddine ${ }^{1}$, Leighton Folkes ${ }^{3}$, Simon Moxon ${ }^{3}$, Ioanna Myrtziou ${ }^{2}$, \\ Susan E. Ozanne ${ }^{4}$, Mandy J. Peffers ${ }^{1}$, Katarzyna Goljanek-Whysall ${ }^{1,5,+}$ and Aphrodite Vasilaki ${ }^{1,+}$
}

Citation: Kanakis, I.; Alameddine, M.; Folkes, L.; Moxon, S.; Myrtziou,

I.; Ozanne, S.E.; Peffers, M.J.;

Goljanek-Whysall, K.; Vasilaki, A.

Small-RNA Sequencing Reveals

Altered Skeletal Muscle microRNAs

and snoRNAs Signatures in

Weanling Male Offspring from

Mouse Dams Fed a Low Protein Diet during Lactation. Cells 2021, 10 ,

1166. https://doi.org/10.3390/

cells10051166

Academic Editor: Giorgio Malpeli

Received: 13 April 2021

Accepted: 9 May 2021

Published: 11 May 2021

Publisher's Note: MDPI stays neutral with regard to jurisdictional claims in published maps and institutional affiliations.

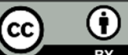

Copyright: $@ 2021$ by the authors. Licensee MDPI, Basel, Switzerland. This article is an open access article distributed under the terms and conditions of the Creative Commons Attribution (CC BY) license (http://creativecommons.org/licenses/by/4.0/).
1 Department of Musculoskeletal \& Ageing Science, Institute of Life Course \& Medical Sciences, Faculty of Health \& Life Sciences, University of Liverpool, Liverpool L7 8TX, UK; M.Alameddine@liverpool.ac.uk (M.A.); peffs@liverpool.ac.uk (M.J.P.); kasia.whysall@nuigalway.ie (K.G.-W.); vasilaki@liverpool.ac.uk (A.V.)

2 Chester Medical School, Faculty of Medicine and Life Sciences, University of Chester, Chester CH2 1BR, UK; i.myrtzioukanaki@chester.ac.uk

3 School of Biological Sciences, Faculty of Science, University of East Anglia, Norwich NR4 7TJ, UK; L.Folkes@uea.ac.uk (L.F.); s.moxon@uea.ac.uk (S.M.)

4 Metabolic Research Laboratories, Wellcome-MRC Institute of Metabolic Science, University of Cambridge, Cambridge CB2 0QQ, UK; seo10@cam.ac.uk

5 Department of Physiology, School of Medicine and REMEDI, CMNHS, NUI Galway, Galway H91 TK33, Ireland

* Correspondence: i.kanakis@chester.ac.uk or i.kanakis@liverpool.ac.uk

+ These authors joint last authorship.

\begin{abstract}
Maternal diet during gestation and lactation affects the development of skeletal muscles in offspring and determines muscle health in later life. In this paper, we describe the association between maternal low protein diet-induced changes in offspring skeletal muscle and the differential expression (DE) of small non-coding RNAs (sncRNAs). We used a mouse model of maternal protein restriction, where dams were fed either a normal $(\mathrm{N}, 20 \%)$ or a low protein $(\mathrm{L}, 8 \%)$ diet during gestation and newborns were cross-fostered to $\mathrm{N}$ or L lactating dams, resulting in the generation of NN, NL and LN offspring groups. Total body and tibialis anterior (TA) weights were decreased in weanling NL male offspring but were not different in the LN group, as compared to NN. However, histological evaluation of TA muscle revealed reduced muscle fibre size in both groups at weaning. Small RNA-sequencing demonstrated DE of multiple miRs, snoRNAs and snRNAs. Bioinformatic analyses of miRs-15a, -34a, -122 and -199a, in combination with known myomiRs, confirmed their implication in key muscle-specific biological processes. This is the first comprehensive report for the DE of sncRNAs in nutrition-associated programming of skeletal muscle development, highlighting the need for further research to unravel the detailed molecular mechanisms.
\end{abstract}

Keywords: maternal protein restriction; muscle development; offspring; microRNAs; sncRNAs; snoRNAs

\section{Introduction}

Intrauterine growth restriction and low birthweight are linked with a predisposition to adverse health consequences in adulthood, including insulin resistance, cardiovascular disease, hypertension and obesity [1]. Therefore, the concept of foetal and perinatal programming of later disease has been developed. Maternal diet during gestation and lactation plays a crucial role in embryonic and post-natal development of the offspring as it is 
the only source of nutrients through the placenta in pregnancy and provides all essential components for neonatal growth during lactation. Poor maternal nutrition during gestation and/or lactation is known to reduce growth and impair muscle development and stem cell activity [2-4], increase fat accretion [5] and alter metabolism [6] in the offspring. Offspring skeletal muscle development is susceptible to maternal nutrient restriction which also results in reduced offspring birth weight caused by decreases in foetal circulating amino acids $[7,8]$. Studies using ovine foetuses and offspring exposed to a poor nutritional environment in utero highlighted deficient muscle growth due to altered myofibre number and composition [9,10]. Further studies using mouse and rat models of maternal protein restriction suggested that this effect on muscle function may be long-lasting throughout the life-course [11-13]. There is also evidence from human studies of low muscle fibre score in elderly men born with low birth weight [14]. However, there is little understanding of the cellular and molecular mechanisms whereby environmental modulation in utero or in early postnatal life may lead to altered development of the musculoskeletal system $[15,16]$.

The epigenetic regulation of skeletal muscle development and ageing has gained significant interest during the last decade $[17,18]$. Small non-coding RNAs (sncRNAs) are a class of epigenetic molecules including microRNAs (miRs), small nucleolar RNAs (snoRNAs) and small nuclear RNAs (snRNAs). These RNA molecules are fully functional, transcribed from DNA without processing for translation into proteins and, thus, act as crucial factors regulating gene expression. All three types of sncRNAs have been implicated in myogenesis and skeletal muscle development and regeneration [19,20]. miRs are non-coding RNAs (ncRNAs), 19-22 nucleotides (nt) in length, that regulate post-transcriptional gene expression and are linked to muscle development, disease and ageing [21,22]. Dietary interventions result in changes in miR expression in muscle [23], suggesting that the effects of diet on muscle may be mediated by miR-regulated changes in gene expression. miRs can simultaneously regulate many genes and signalling pathways, and therefore potentially physiological and pathological processes. It has been suggested that not all miRs are ubiquitously expressed, but many miRs are expressed in a tissue-specific manner $[24,25]$. There is a class of miRs in which their expression is directly associated with striated muscle, known as myomiRs [26], including miR-1, miR-133a, miR-133b, miR-206, miR-208b, miR-486 and miR-499 [27-30]. Although myomiRs have been extensively studied, other miRs may also be involved in regulating skeletal muscle development.

Ribosomes are considered as the cellular converters of the genetic codes, embedded in messenger RNAs, converting these codes into proteins [31]. It is known that ribosomal RNAs (rRNA) are post-transcriptionally modified via 2'O-ribose methylation or pseudouridylation [32]. These site-specific covalent modifications regulate the translational process. snoRNAs are mainly intron-derived, 50-250 nt long ncRNAs with high expression levels accumulating in the nucleolus. They are classified as C/D box snoRNAs (SNORDs), which are responsible for 2'-O-methylation of rRNAs, or H/ACA box snoRNAs (SNORAs), which guide pseudouridylation of nucleotides [33,34]. However, approximately half of human snoRNAs have no predictable rRNA targets, and numerous snoRNAs have been associated with diseases [35,36] that show no defects in rRNAs [37]. On the other hand, snRNAs have an average size of $150 \mathrm{nt}$, which localise in the nucleus and mainly control intron splicing [38]. snRNAs are present as ribonucleoprotein particles (snRNPs) forming a large complex (spliceosome) which mediate splicing by attachment to the unspliced primary RNA transcripts [39-41]. Despite the vital roles described, very little is known for the specific functions of snoRNAs and snRNAs in skeletal muscle physiology.

In this study, we aimed to identify miRs, snoRNAs and snRNAs that may regulate reduced skeletal muscle growth in the offspring due to maternal protein undernutrition during pregnancy or during lactation. We utilised a mouse model of maternal protein restriction and analysed small RNA-seq data derived from skeletal muscle of 21 days (21d) male offspring to determine sncRNAs that are differentially expressed at weaning. 
Furthermore, we validated some of the observed differentially expressed (DE) miRs by qPCR and performed an in silico bioinformatic analysis to unravel which signalling pathways and cellular functions may be affected by these miRs.

\section{Materials and Methods}

\subsection{Animals}

B6.Cg-Tg(Thy1-YFP)16Jrs/J mice, expressing yellow fluorescent protein (YFP) only in neuronal cells (Jackson Laboratory; stock number 003709), were used in this study. Mice were housed in individually vented cages maintained at $21 \pm 2{ }^{\circ} \mathrm{C}$ on a $12 \mathrm{~h} \mathrm{light/dark}$ cycle. All experimental protocols were performed according to the UK Animals (Scientific Procedures) Act 1986 regulations and obtained ethical approval from the University of Liverpool Animal Welfare Ethical Review Board (AWERB). Animal use followed the 3Rs guidelines. Mice were kept at the Biomedical Services Unit (BSU) of the University of Liverpool and monitored daily for any health and welfare issues.

\subsection{Experimental Groups}

Mice were fed ad libitum with solid food pellets containing normal protein proportion (N, 20\% crude protein; Special Diet Services, UK; code 824226) or low protein proportion (L, 8\% crude protein; Special Diet Services, UK; code 824248) with isocaloric value. Eight-week-old nulliparous female mice were fed on either $\mathrm{N}$ or L protein diet for two weeks before mating with age-matched males on $\mathrm{N}$ diet. The pregnant mice were kept on the same diet throughout gestation (19-21 days). Within $24 \mathrm{~h}$ after birth, male pups from $\mathrm{N}$ dams were cross-fostered with other $\mathrm{N}$ dams (NN group, $n=5$ ) or L dams (NL group, $n=5$ ) that were maintained under the same experimental conditions, but fed with $\mathrm{L}$ diet two weeks prior to mating and during gestation and lactation (21 days), whilst pups from $\mathrm{L}$ dams were transferred to $\mathrm{N}$ dams for lactation $(\mathrm{LN}, n=5)$. Suckling pup numbers were kept the same for all dams during the $21 \mathrm{~d}$ lactation period ( $n=5 \mathrm{pups}$ ) to avoid confounding issues of litter size difference (Figure 1A). Male offspring were culled at the end of lactation by $\mathrm{CO}_{2}$ euthanasia and weighed and tibialis anterior (TA) skeletal muscles were immediately dissected and weighed.

\subsection{Skeletal Muscle Histology}

To characterise the skeletal muscle phenotype, TA muscles ( $n=4$ /group) were mounted directly on a cork disk surrounded with Cryomatrix (Thermo Scientific ${ }^{\mathrm{TM}}$, UK), immersed in liquid nitrogen-frozen isopentane (Sigma Aldrich, UK) for cryoprotection and stored at $-80^{\circ} \mathrm{C}$ until cryosectioning. TA muscles were placed at $-20^{\circ} \mathrm{C}$ for at least 30 min prior to cryosectioning. Transverse sections $(10 \mu \mathrm{m})$ were cut using a Leica cryotome and collected on Superfrost glass slides (Thermo Scientific ${ }^{\mathrm{TM}}$, UK). Sections were washed with PBS for 10 min before staining with 1:1000 dilution of rhodamine wheat germ agglutinin (WGA; $5 \mu \mathrm{g} / \mathrm{mL}$; Vector Laboratories, UK) for $10 \mathrm{~min}$, mounted in antifade medium (Vector Laboratories, UK) and were visualised with an Axio Scan.Z1 slide scanner (Zeiss, UK). Minimum Feret's diameter (MFD) was measured using ImageJ software (U.S. National Institutes of Health, USA).

\subsection{RNA Isolation, Library Preparation for Small RNA-Seq and Sequencing}

TA skeletal muscles were ground using a mortar and pestle and liquid nitrogen. Total RNA was isolated and purified by the mirVanaTM kit (Thermo Scientific ${ }^{\mathrm{TM}}$, UK) using the manufacturer's protocols. Samples were processed with Clip-Clap ${ }^{\mathrm{TM}}$ Acid Pyrophosphatase (Cambio, UK) prior to the library preparation to remove any $5^{\prime}$ cap structures, and size selected using a range 120-300 bp including adapters ensuring the unbiased identification of sncRNAs. Preparation of a small RNA-Seq library from submitted total RNA sample was performed using the NEBNext ${ }^{\circledR}$ small RNA library preparation kit (New England Biolabs, UK). Small-RNA sequencing was conducted using the Illumina HiSeq 4000 
(Illumina, San Diego, CA, USA) at $2 \times 150$-base pair (bp) paired-end sequencing, generating data from $>280 \mathrm{M}$ clusters per lane. Sequencing was performed at the Centre for Genomic Research, University of Liverpool (https://www.liverpool.ac.uk/genomic-research/, accessed on 9 May 2021).

\subsection{Small RNA-Seq Data Processing}

Mouse (mmu) mature miR sequences were downloaded from miRBase (v22.1) [42] in FASTA format. For the alignment of sRNA reads, the FASTA format miRBase sequence files were made non-redundant using java code and Uracil bases (U) were changed to thymine (T). Small RNA reads were converted from FASTQ to FASTA format and then processed to trim sequencing adaptors using the Perl script "Remove adaptors" [43], recognising the first 8 bases of the adapter sequence. The processed reads were then aligned to the processed mature miR sequences allowing zero mismatches using PatMaN [44] (parameters: -s -e 0 -g 0 and -s -e 1 -g 0 ). Custom java code was used to parse the alignment files and generate an aligned read count table across all samples. The DESeq2 [45] method within iDEP [46] (version 91) was used for normalisation of counts between samples and calling differentially expressed (DE) miRs using default settings.

To obtain sncRNA reference sequences, the Mus musculus (Genome Assembly GRCm38) annotation in gff3 format was downloaded from Ensemble [47] (release-96) from which all records representing a non-coding RNA gene with a snoRNA biotype were extracted and converted into bed format. The processed annotation file in bed format along with the Mus musculus genome top level assembly (Ensemble release-96, GRCm38) was used with the function "getfasta" within the bedtools software [48] (parameters: -s name) to extract snoRNA sequences in FASTA format. The FASTA format snoRNA sequence file was then made non-redundant using java code. The processed sRNA reads were then aligned to the snoRNA sequences, aligned read count tables were generated and differential expression analysis was performed as described above. Java code used for sequencing processing along with count matrices are available online at https://github.com/lf-bioinformatics/sRNA-code, accessed on 9 May 2021.

Data were assessed using pairwise comparisons, while correlation heatmaps and principal component analysis (PCA) plots were visualised using the Clustvis web tool [49]. DE miRs were extracted by applying the threshold of false discovery rate (FDR) adjusted $p$-values $<0.05$ (FDR <0.05), generated using the Benjamini-Hochberg method [50] and an absolute value of $\log 2$ fold change of $1.0(\mid \log 2 \mathrm{FCl}>1$, equating to a 2-fold change) in the first instance; thresholds of FDR $<0.001$ and $\mid \log 2 \mathrm{FCl}>1.3$ (equating to a 2.5-fold change) and FDR $<0.05$ and $\mid \log 2 \mathrm{FCl}>1.3$ were applied when narrowing the $\mathrm{DE} \mathrm{miR}$ and snoRNAs ranges, respectively.

\subsection{Quantitative Polymerase Chain Reaction ( $q P C R$ )}

For miR expression analysis, total RNA was isolated and purified using the mirVanaTM kit (Thermo Fischer, UK), reverse transcription of total RNA containing miRs was performed with miScript II RT kit (Qiagen, UK). qPCR was performed on a RotorGene ${ }^{\mathrm{TM}}$ 6000 (Corbett Research) instrument in a $20 \mu \mathrm{L}$ reaction mixture; qPCR conditions were: 95 ${ }^{\circ} \mathrm{C}$ for $30 \mathrm{~s}, 55^{\circ} \mathrm{C}$ for $30 \mathrm{~s}$ and $72{ }^{\circ} \mathrm{C}$ for $30 \mathrm{~s}$ (40 cycles) using a hot start step of $95{ }^{\circ} \mathrm{C}$ for 15 s. Specific primers for miR-15a, $-34 a,-125 b,-199$ and -206 (Table S1) were used for the qPCR utilising RNU6 as the reference gene [51,52]. The results were analysed using the modified delta CT method [53].

\subsection{Bioinformatic Pathway Analysis of DE miRs and miR:Target Prediction}

Potential biological connections of all DE miRs were identified using Ingenuity Pathway Analysis (IPA) (IPA, Qiagen Redwood City, CA, USA) "Core Analysis". Putative miR-target gene prediction was performed by uploading DE miR data into the MicroRNA Target Filter module within IPA. This module combines experimentally validated 
databases, peer-reviewed publications and target gene predictions using TargetScan [54] to identify miR-mRNA interactions and creates biological networks describing functional associations. For each core analysis, "diseases and bio-functions" were queried. In addition, to predict gene targets of the selected miRs, the miRWalk on-line tool [55] was utilised by searching through four databases: miRWalk, TargetScan, miRDB [56] and MiRTarBase [57]. Cytoscape v3.7.2 [58] software was used to build the interaction networks between predicted targets and selected miRs as well as to determine the biological roles of the target mRNAs utilising Gene Ontology (GO) terms of biological process and molecular functions using the ClueGO plug-in [59]. KEGG pathway analysis [60] was performed to determine the implication of the predicted gene targets in biological pathways. $p<0.05$ was considered to indicate a statistically significant result.

\subsection{Statistical Analysis}

All data were analysed with GraphPad Prism 6 software and expressed as the mean \pm SD. Data sets were tested for Gaussian distribution with the D'Agostino-Pearson normality test. Comparisons between the NN and NL groups were performed by unpaired Student's $t$-test or Mann-Whitney U test. Bioinformatic analyses statistics were performed with built-in packages in IPA and Cytoscape. The $p$ values in GO analysis were calculated with Fisher's Exact test corrected with Bonferroni post hoc test in Cytoscape. In all cases, $p$ values less than 0.05 were considered statistically significant.

\section{Results}

\subsection{Skeletal Muscle Phenotypic Characterisation of Male Offspring}

Male offspring were collected at weaning (21d) and the size and total body weight were compared using the $\mathrm{NN}$ group as control ( $\mathrm{NN}$ vs. $\mathrm{NL}$ and $\mathrm{NN}$ vs. $\mathrm{LN}$ ). Our data indicate that mice born from dams maintained on a normal protein diet but fed postnatally by a foster dam maintained on a low diet (NL) showed significant reductions in body size (Figure 1B) and total body weight (Figure 1C) at 21 days compared to NN male mice. On the contrary, neonates born from dams on a low protein diet but were fed by dams on normal protein diet during lactation (LN) appeared to have equal mean size and total body weight to the NN group at weaning, as shown in Figure 1B,C. It is important to note that the total body weight of P0 (day of birth) neonates born from dams on low protein diet $(\mathrm{L})$ was significantly lower than pups born from dams on normal protein $\operatorname{diet}(\mathrm{N})$ (Figure S1A,B).

TA muscle weight index (expressing the net TA weight per total body weight of each pup) for NL offspring was lower compared to NN offspring, demonstrating a profound effect of maternal low protein consumption during lactation which was not observed in muscles from LN males (Figure 1D). To test if muscle size was associated with muscle fibre size, we compared the MFD in WGA stained cryosections from fibres in the midshaft of the TA muscle. We found that the MFD of TA muscle fibres from the NL and LN groups was significantly lower than the NN group (Figure 1E,F). 


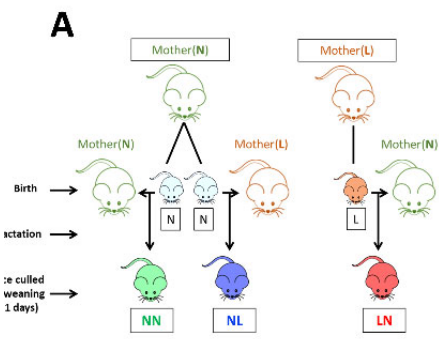

B

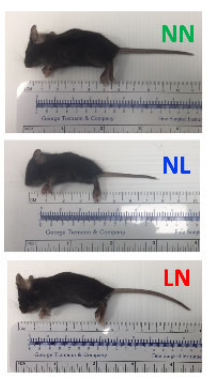

D

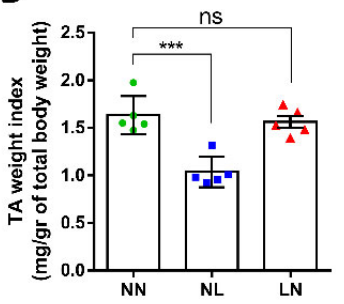

$\mathbf{E}$

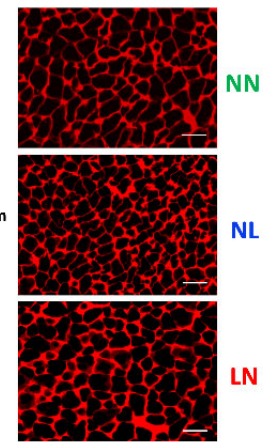

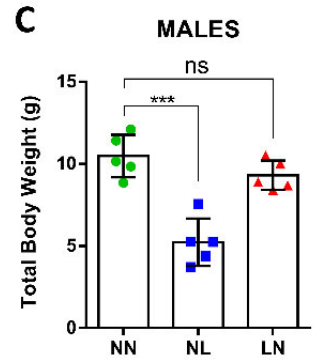

F TA skeletal muscle

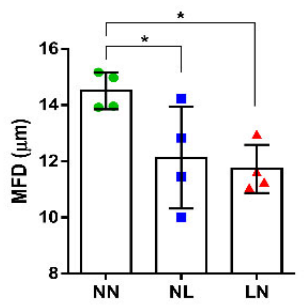

Figure 1. Experimental design and skeletal muscle morphology. Illustrative description of the experimental design of the study (A). Male 21d offspring lactated by dams on low protein diet (NL) were smaller in size (B) and had lower total body (C) and TA muscle/body weight index (D) compared to the NN group, while offspring lactated by dams on normal protein diet (LN) did not differ from NN control group. Histological assessment revealed that TA myofibre size was decreased in both NL and LN groups, as shown in representative images $(\mathbf{E})$ and measurements of muscle fibre minimum Feret's diameter (MFD) (F). All data are presented as mean \pm SD. ${ }^{*} p<0.05,{ }^{* * *} p<$ 0.001 , ns, non significant. Scale bar: $100 \mu \mathrm{m}$.

\subsection{Differential Expression Analysis of miRs in TA Skeletal Muscle}

Small RNA-seq was performed using total RNA isolated from TA muscles of male offspring at weaning. All RNA samples (500 ng) were of high purity and integrity with RNA Integrity Number (RIN) $>7$ as assessed on the Agilent 2100 Bioanalyzer system (Agilent Technologies Ltd., UK). An average of 14.18 million total reads for NN, 14.72 million for NL and 14.05 million for LN group was generated, of which $13.28 \%, 3.65 \%$ and $13.96 \%$ were mature miRs with mapped reads of 2.05 million, 0.51 million and 1.99 million, respectively. Normalised reads were used to estimate small RNA transcript expression of all samples, aiming to identify the most abundant sncRNAs. In total, 796 mature miRs were detected (Figure $2 \mathrm{~A}$ ). Only miRs with at least 10 raw reads in $\geq 60 \%$ of the samples in each group ( $\geq 3$ samples/group) were considered measurable, and, after filtering with $\mathrm{FDR} \leq 0.05$ and $|\log 2 \mathrm{FC}|>1$ (Fold Change), $179 \mathrm{miRs}$ were extracted (Figure 2B). The DE of common miRs among the three groups, 145 in total, was statistically significant when $\mathrm{NN}$ and NL groups were compared, while no differences in miR expression levels were found between NN and LN groups. The only exception was miR-122 expression, which was slightly increased in LN group in comparison with $\mathrm{NN}(p=0.046$, Table S2).

To reduce the high number of DE mature miRs in order to have a range of the most significant common miRs with the highest potency and a possible biological role, we applied an additional stricter filter of FDR $<0.001$ and $\mid \log 2 \mathrm{FCl}>1.3$ ( $>2.5$-fold change), which resulted in 92 miRs (Table S2). A PCA of NN, NL and LN samples using the DE of these 92 miRs indicated a significant difference of the NL group against the NN/LN groups (Figure 2C). The samples of the latter groups were overlapped in the PCA plot, indicating similarity in miR expression (Figure 2C). Finally, a heatmap revealed a clear difference in the distribution of these 92 common miRs between the NN/LN and NL 
groups of male mice (Figure 2D). Notably, two groups of samples were distinguishable: the DE of miRs from TA muscles of NL samples were assembled in one cluster, while NN and LN samples were presented as a combined cluster, indicating similar miR expression levels (Figure 2D).
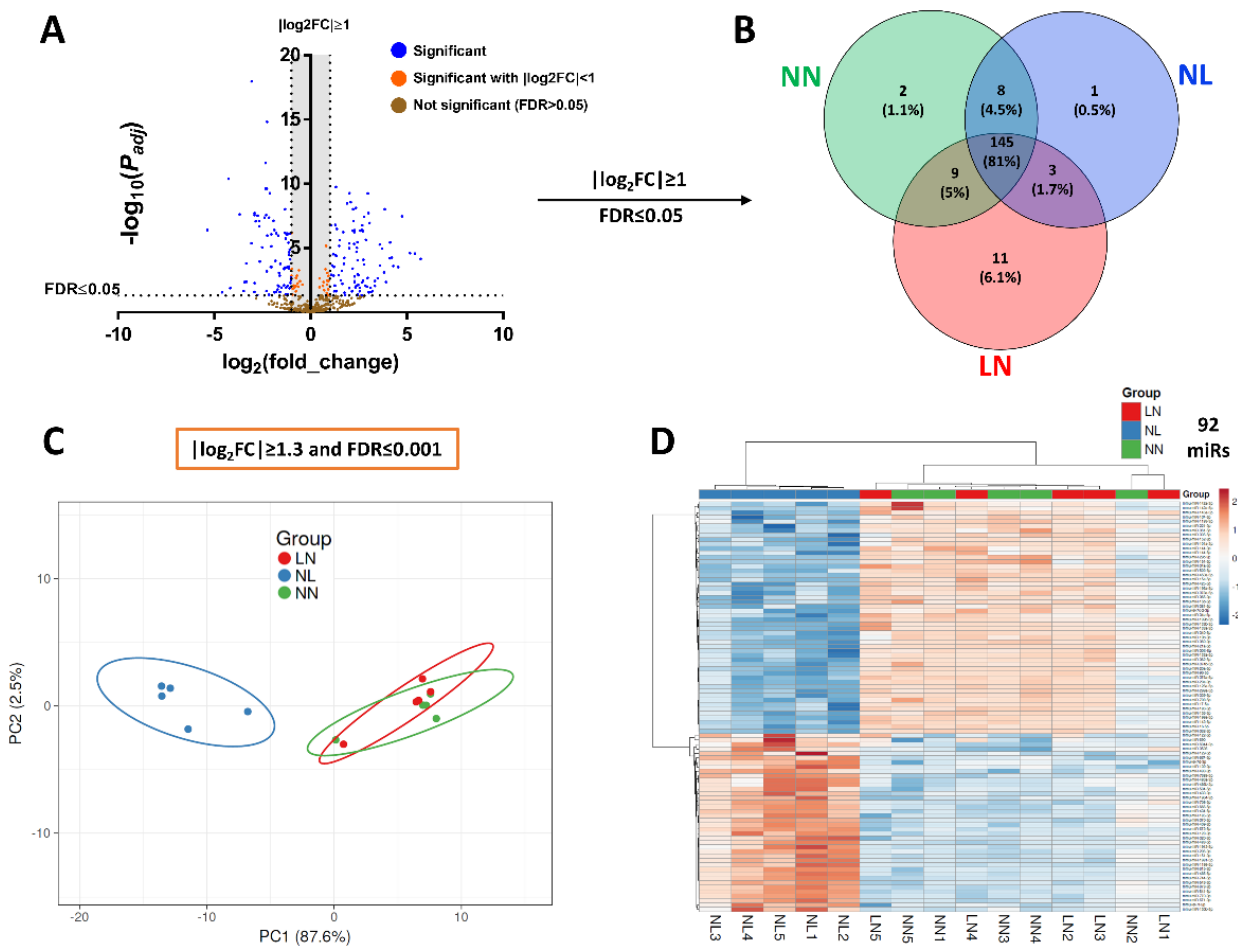

Figure 2. Differential expression of miRs. Small RNA-seq data analysis ( $n=5 /$ group) showed differential expression (DE) of numerous miRs, illustrated by Volcano plot (A). After filtering with FDR $\leq 0.05$ and $\mid \log 2 \mathrm{FCl} \geq 1$, there were 179 remaining DE miRs, where 145 were common among NN, $\mathrm{NL}$ and LN groups (B). Application of additional and stricter filters, FDR $\leq 0.001$ and $\mid \log 2 \mathrm{FCl} \geq$ 1.3, narrowed the number of common DE miRs to 92 (see Table S2); PCA analysis indicated a profound difference between NN and NL groups (C), whilst NN and LN were mapped together. These differences were also reflected in expression heatmap of these $92 \mathrm{miRs}$ (D). The colour of each entry is determined by the number of reads, ranging from red (positive values) to blue (negative values).

\subsection{Ingenuity Pathway Analysis for All DE miRs}

To explore the potential biological associations of the $92 \mathrm{DE}$ miRs in TA skeletal muscles from NL versus NN/LN male offspring, we undertook an IPA "Core Analysis". Networks were generated based on information from Ingenuity Pathway Knowledge Database. Significant cellular functions determined by the DE miRs and predicted gene targets included "morphology of muscle cells" ( $p=0.017)$ and "differentiation of muscle cells" ( $p$ $=0.008)$. The most interesting diseases associated with the constructed networks were "disarray of muscle cells" ( $p=0.022)$ and "dystrophy of muscle" $\left(p=1.11 \times 10^{-6}\right)$ (Figure $3 \mathrm{~A})$. The top scoring network identified was "Organismal Injury and Abnormalities", with $p$ value ranging between $4.87 \times 10^{-2}$ and $7.41 \times 10^{-6}$ and involving $62 \mathrm{DE}$ miRs. Finally, IPA revealed two statistically significant GOs associated with the DE miRs, namely "apoptosis of muscle cells" $\left(p=5.58 \times 10^{-8}\right)$ and "proliferation of muscle cells" $(p=1.36 \times$ $10^{-10}$ ) (Figure 3B), as well as statistically significant GOs associated with predicted gene targets, e.g., "muscular hypertrophy" $\left(p=8.51 \times 10^{-8}\right)$ and "muscle contraction" $(p=1.5 \times$ $10^{-6}$ ) (Figure 3C). A full list of statistically significant GOs associated with muscle is provided in Table S3. 

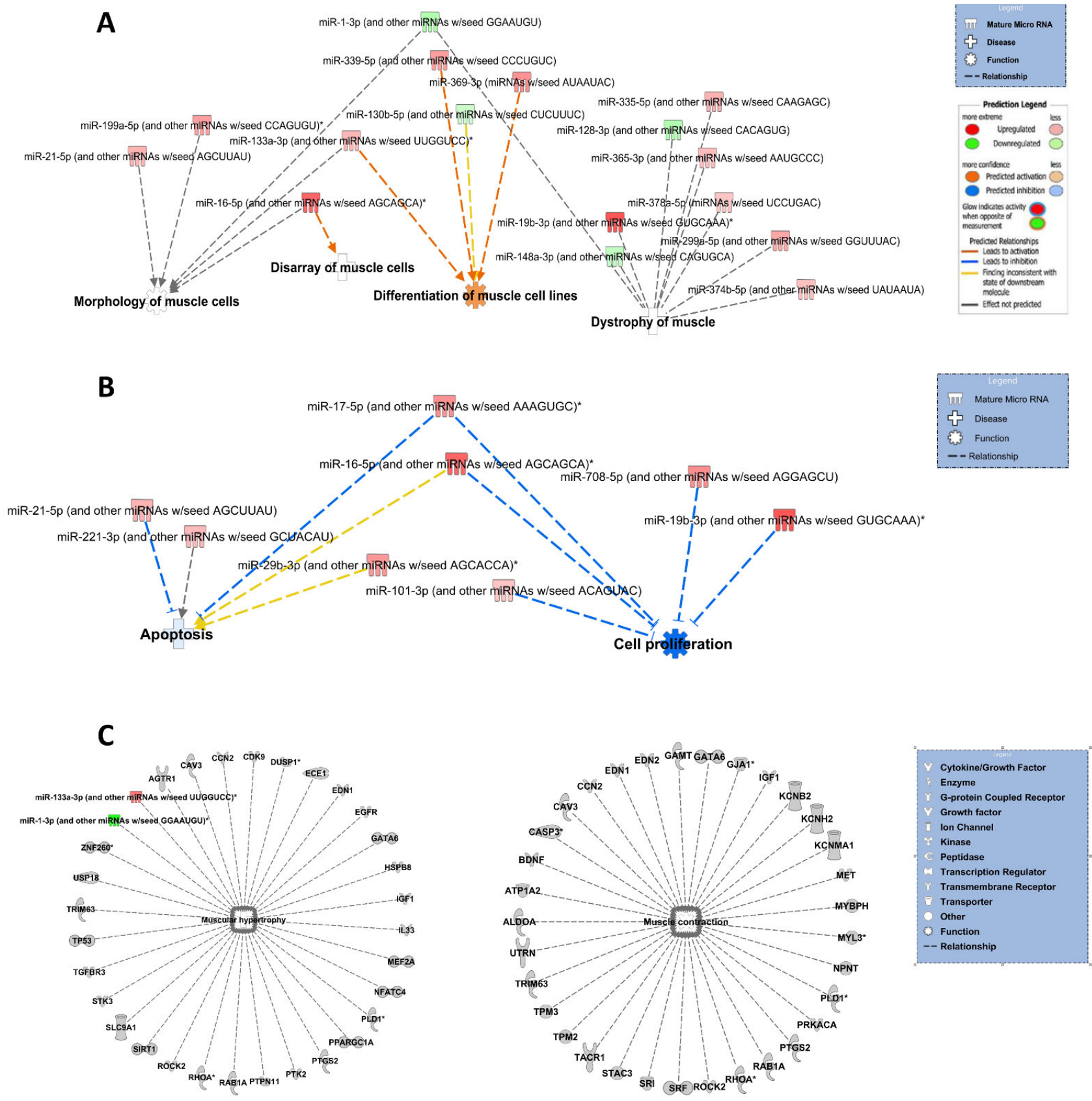

Figure 3. Bioinformatic analyses of differentially expressed miRs. Ingenuity Pathway Analysis representation of functions using DE miRs between NN/LN and NL male groups. Differentiation, morphology and disarray of muscle cells were identified by IPA as the predominant cellular functions and dystrophy of muscle as the most relevant disease which were correlated with the 92 differentially expressed miRs (A). Top GO terms identified in miR IPA core analysis were apoptosis and cell proliferation which relate to specific miRs (B). Muscle hypertrophy and contraction were the top GO terms associated with predicted gene targets of DE miRs (C). Figures are graphical representations of miRs identified in our data in their respective networks. Red nodes correspond to upregulated gene expression in NN/LN, and green nodes downregulation. Intensity of colour is related to higher fold-change. Legends to the main features in the networks are shown. The function colour is dependent on whether it is predicted to be activated or inhibited.

\subsection{Selection and Validation of miRs from Small RNA-Seq}

The next step was to select miRs which demonstrated a strong difference, in terms of both fold change DE and FDR, and among the NN, LN and NL groups of male mice which 
could have an impact of skeletal muscle development. We focused on four miRs: miR-15a, $-34 a,-122$ and $-199 a$. These specific miRs were selected based on our current work, the level of DE and following a literature search of the DE genes.

Among the 92 mature miRs with statistically significant DE, 42 were found with lower expression levels in NN/LN as compared to the NL group, including miR-122, while 50 were upregulated in NN/LN, including miR-15a, -34a and -199a (Figure 4A). Next, we tested if the selected miRs (miR-122, miR-15a, miR-34a and miR-199a) in combination with established and well-studied muscle-specific myomiRs [26], such as miR-1, -133a, -133b, $206,-208 b,-486$ and -499 , known regulators of muscle cell proliferation and differentiation, can form a group of miRs able to profoundly distinguish the two groups of mice based on their expression levels in TA skeletal muscle. Indeed, a miR expression levels-generated heatmap, including these miRs, showed a clear DE of this group of miRs, highlighting the significant phenotypic difference in skeletal muscles affected by maternal protein restriction during lactation in NL males (Figure 4B). Finally, qPCR analysis was used to validate the miR-seq data. We found that miR-122 was expressed at higher levels in NL (Figure $4 \mathrm{C}$ ), but not $\mathrm{LN}$, a finding that contradicts the sequence data. miRs-15a, $-199 \mathrm{a}$ and 34a expression levels were significantly lower in the NL mice, as shown in Figure 4D-F, respectively. These results are in agreement with miR-seq, while miR-206 expression levels were higher in NN than in NL and LN, which contradicts the sequence data (Figure $4 \mathrm{G}$ ). In addition, miR-34a expression levels were found lower by qPCR in LN as compared to $\mathrm{NN}$, a result which also opposes the miR-seq data (Figure $4 \mathrm{~B}, \mathrm{~F}$ ).

A

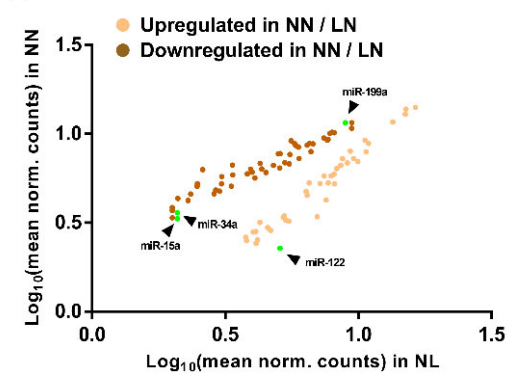

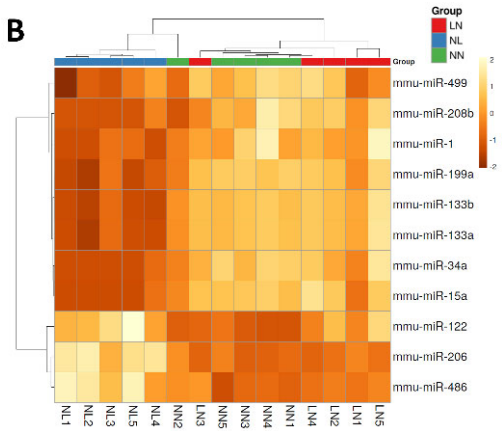

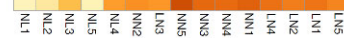

C

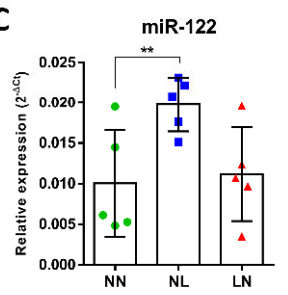

D

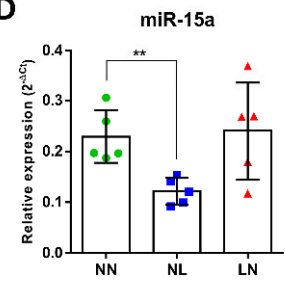

E

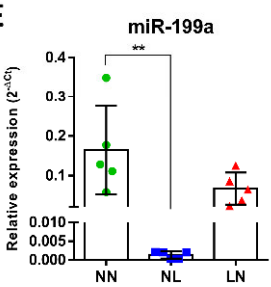

$\mathbf{F}$

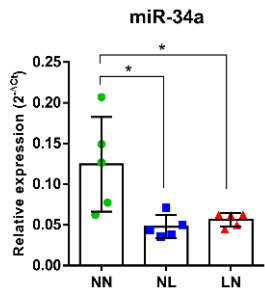

G

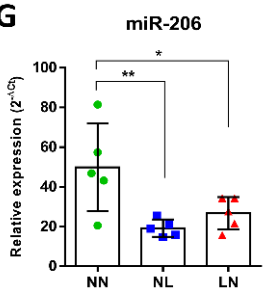

Figure 4. Validation of selected differentially expressed miRs by qPCR. Among the 92 miRs with DE between NN/LN and NL as determined by small RNA-seq, miR-122 expression was higher in NL, whereas miR-15a, -34a and -199a were found upregulated in NN and LN (A). The combination of these four miRs with known myomiRs revealed an interactive set of skeletal muscle-associated 
miRs in which the degree of DE between NN/LN and NL is very strong, as illustrated by the expression heatmap (B). The colour of each entry is determined by the number of reads, ranging from yellow (negative values) to brown (positive values). To confirm the expression levels, validation by qPCR showed increased expression in NL, but not in LN, for miR-122 (C). The results were similar between small RNA-seq and qPCR for miR-15a (D) and miR-199a (E), while for miR-34a (F) and miR-206 (G) were different between the two detection systems. ${ }^{*} p<0.05,{ }^{* *} p<0.01$.

\subsection{Selected miRs-15a, -34a, -122 and -199a Bioinformatic Pathway Analysis}

Based on the observations and data described above, we performed a bioinformatic analysis to explore the possible molecular pathways and cellular functions that the predicted target genes of our selected mature miRs could be implicated in. Using a combination of strict parameters, i.e., seed length range 16-21 at the 3'-UTR site, binding $p$ value of 1 and showing only the statistically significant mRNAs which occurred in all three miR databases (TargetScan, miRDB and MiRTarBase), we identified 70 predicted target genes in total for the four selected miRs (Figure 5A). GO analysis using the GlueGO plug-in of the Cytoscape software and including biological process, cellular functions and KEGG pathways revealed an interactive molecular network, as shown in Figure 5B. The six GO terms with the lower $p$ values after Bonferroni corrections were: "cerebellum morphogenesis" ( $p=0.0017)$, "embryonic pattern specification" ( $p=0.0015)$, "regulation of striated muscle cell apoptotic process" $\left(p=4.2 \times 10^{-4}\right)$, "embryonic cranial skeleton morphogene$\operatorname{sis}^{\prime \prime}\left(p=2.21 \times 10^{-4}\right)$, "positive regulation of cellular response to insulin stimulus" ( $p=5.13$ $\left.\times 10^{-5}\right)$ and "regulation of histone methylation" $\left(p=4.41 \times 10^{-5}\right)($ Figure 5C). 

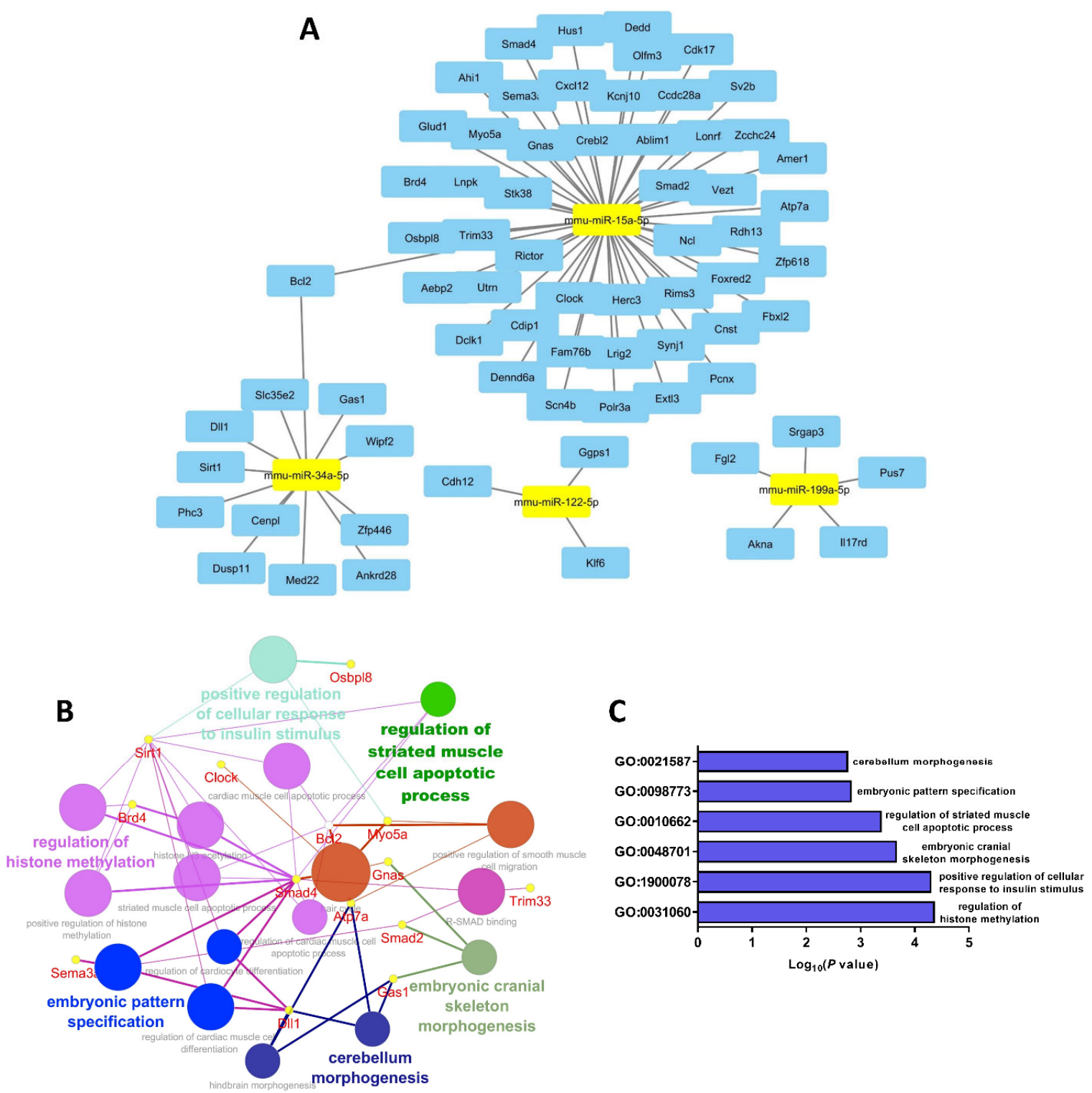

Figure 5. Bioinformatic network of selected miRs predicted gene targets. Target analysis of miR-15a, $-34 a,-199 a$ and -122 by Cytoscape showed numerous (70) predicted gene targets (A). KEGG pathway analysis of the 70 predicted genes indicated that they could interact with each other forming a molecular network that can regulate significant as well as diverse developmental processes. Small circles represent a range of $p$ value of $1.5-1.7 \times 10^{-3}$, moderate circles $2.2-4.2 \times 10^{-5}$ and large circles 4.4-5.1 $\times 10^{-5}$ after Bonferroni correction (B). The most significant GO showing statistical significance were cerebellum morphogenesis, embryonic pattern specification, regulation of striated muscle cell apoptotic process, embryonic cranial skeleton morphogenesis, positive regulation of cellular response to insulin stimulus and regulation of histone methylation (C).

Furthermore, to assess if our selected miRs interact with muscle-specific myomiRs, we used an in silico approach via Cytoscape, which revealed that this set of miRs appear to share some common gene targets forming an interrelated network (Figure S2A). GO analysis showed that this combination of miRs (selected plus myomiRs) were involved in important developmental stages of the musculoskeletal system including motor neuron axon guidance and negative regulation of muscle tissue development (Figure S2B,C). 


\subsection{Analysis of DE snoRNAs and snRNAs in TA Skeletal Muscle}

We further analysed the small RNA-seq datasets to identify the DE snoRNA and snRNAs from TA skeletal muscles. Averages of 14.18 million total reads for NN, 14.72 million for NL and 14.05 million for LN group were generated, of which $12.77 \%, 14.95 \%$ and $10.88 \%$ were snoRNAs and snRNAs with mapped reads of 1.80 million, 2.22 million and 1.51 million, respectively. We detected 514 snoRNA and snRNAs in total, of which 95 were considered statistically significant, having FDR $\leq 0.05$ and $\mid \log 2 \mathrm{FCl}>1.3$ (Figure 6A). It is worth noticing that, of these 343 sncRNAs, 2 were identified as snRNAs (RNU3a and RNU73b), $39 \mathrm{H} / \mathrm{ACA}$ box snoRNAs (SNORA), $80 \mathrm{C} / \mathrm{D}$ box snoRNAs (SNORDs), 2 small Cajal body-specific RNAs (scaRNAs) and 220 uncharacterised (Figure 5B). After FDR and $\log 2$ FC filter application, we used 2 snRNAs, 11 SNORAs, 26 SNORDs and 56 uncharacterised sncRNAs (Figure 6B and Table S4). The analysis of normalised counts showed that Rnu3a was highly expressed in the TA muscles from NL group in comparison with either NN or LN. On the contrary, Rnu73a expression was significantly increased in NN and LN mice (Figure 6C). PCA analysis using DE SNORAs and SNORDs showed that NL samples were clustered tightly while NN and LN samples were grouped (Figure $6 \mathrm{D})$. The heatmap representing DE revealed a clear difference in the distribution of SNORAs and SNORDs between the NN/LN and NL groups of male mice (Figure 6E).

A

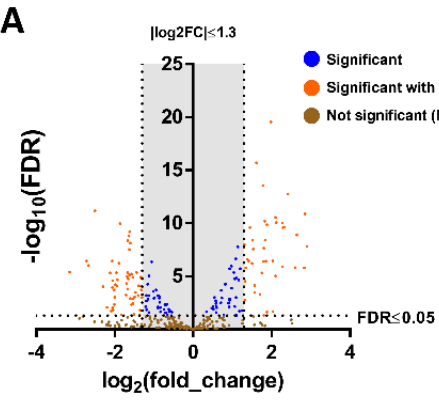

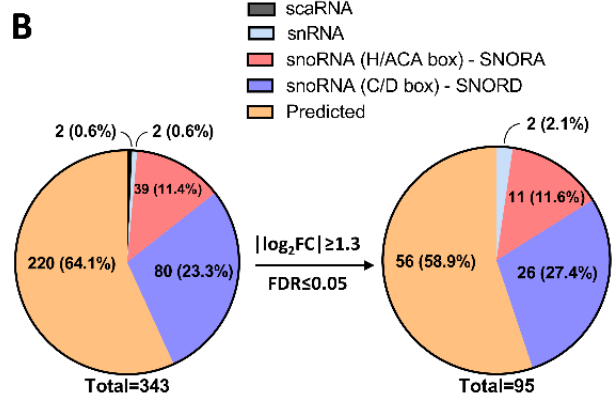

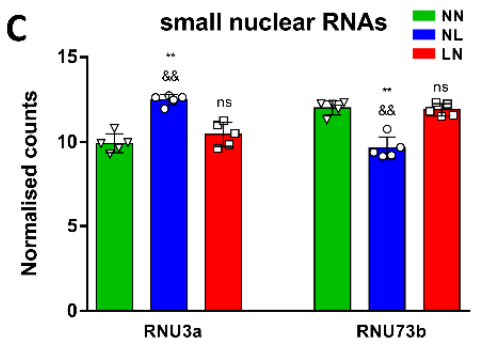

D
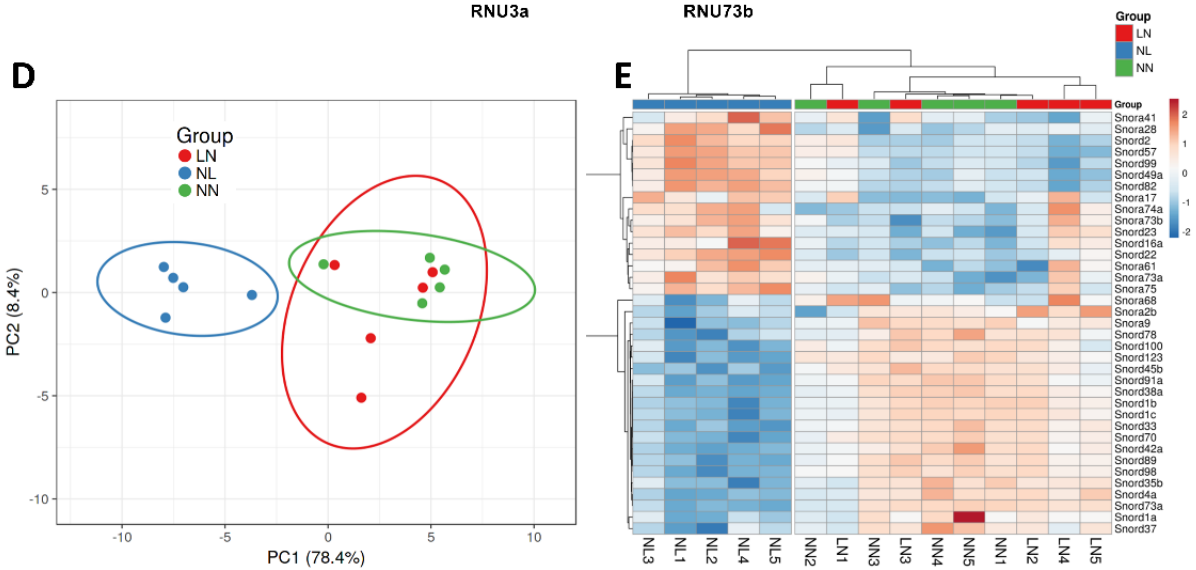

Figure 6. Bioinformatic analyses of differentially expressed sn/snoRNAs. Small RNA-seq data analysis revealed DE of multiple snRNAs and snoRNAs, as shown in the Volcano plot (A). Filters application of FDR $\leq 0.05$ and $\mid \log 2 \mathrm{FCl} \geq 1.3$ 
indicated the residual 95 DE sncRNAs, of which 56 were uncharacterised (B). The expression levels of snRNAs showed statistically significant upregulation of RNU3a and downregulation of RNU73b in NL group in comparison with NN and LN groups (C). PCA analysis demonstrated a profound difference between NN and NL groups (D), whilst NN and LN were clustered together. DE expression levels of SNORAs and SNORDs were plotted as heatmap suggesting distinct clusters of NN/LN and NL samples (E). The colour of each entry is determined by the number of reads, ranging from blue (negative values) to red (positive values). ${ }^{* *} p<0.01$ as compared to $\mathrm{NN}$, \&\& $p<0.01$ as compared to LN; ns, not significant.

\section{Discussion}

In this study, we report that maternal protein undernutrition during lactation (NL) causes a significant decrease in body mass, skeletal muscle weight and muscle fibre size in male offspring at weaning. This phenotype was accompanied by a very strong DE of numerous miRs as well as snRNAs and snoRNAs between NN and NL groups of mice. We also found that, among the plethora of altered miRs, there are four previously unreported DE miRs which strongly correlate with muscle-specific myomiRs and in silico bioinformatic analyses, implicating them in important developmental processes of the musculoskeletal system. Additionally, we demonstrated that mice born from dams fed a low protein diet during gestation but exposed to normal amounts of protein during lactation (LN) have a similar growth to the NN offspring with no apparent differences in body size, body weight or muscle weight at weaning. However, despite the lack of difference in weight, the TA myofibre size in these mice was reduced, which is similar to the reduction observed in the TA myofibres of the NL offspring.

There is growing evidence that the early-life nutritional environment, both in utero and in the early postnatal period, can have persistent long-lasting effects on later life health. Based on the developmental programming concept, this intergenerational effect can lead to high risk of obesity, type 2 diabetes and cardiovascular diseases [1,61]. Skeletal muscle is characterised by plasticity and is highly adaptive to nutritional changes by altering size, metabolic rates and functional properties. It is also affected in response to maternal nutritional imbalance by changes in offspring phenotype [10,62-64]. During foetal development, undernutrition can determine muscle fibre formation and growth in the offspring by suppression of muscle development associated gene expression [65]. Pregnancy and lactation are considered as critical periods, and it is evident that nutrient restriction before weaning may cause permanent changes in skeletal muscle [66]. Consistently, our results demonstrate that maternal low protein intake during lactation results in lower total body and skeletal muscle weight in NL male offspring. The result that pups at birth were found different in size and weight (depending on maternal protein diet during gestation) is in accordance with previous reports [67]. It appears that maternal diet during lactation can modify neonatal growth, since the LN group showed accelerated catch up growth and reached the same size and weight with the NN group at weaning, but not muscle fibre size. On the other hand, although P0 pups from mothers on normal protein diet are bigger, maternal low protein diet during lactation constrains their growth, resulting in smaller size of both total body and muscle fibres. During postnatal developmental stages, skeletal muscle growth is attained by muscle fibre size increase, and not muscle fibre number, mainly by satellite cells proliferation and myoblasts fusion [68], and musclespecific net protein hypertrophic increase until 3 weeks of age [69]. Our results reveal that maternal low protein intake during pregnancy and/or lactation results in decreased size of myofibres, as assessed by fibre size measurements, consistent with other studies in rat [63]. This suggests that gestational as well as lactational protein feeding determines the skeletal muscle fibre size. This is crucial, since the myofibre number is still growing shortly after birth in rodents [70-72]. However, most studies have mainly focused on maternal high-fat diet [73] and cafeteria diets [74,75], but little is known about the mechanisms of low protein diet and how it affects muscle development in the offspring.

Although myogenesis is extensively studied, the focus has been limited to transcription factors and gene regulation. During the last decade, myomiRs have triggered the attention of many studies and next generation sequencing analyses have confirmed that 
myomiRs are abundant in muscle tissue [30]. MyomiRs are expressed in both cardiac and skeletal muscle with the exception of miR-206 [27], which is skeletal muscle-specific. Other studies have reported that some myomiRs are also expressed in other tissues at low levels, but it is accepted that their main roles are still in muscle [25]. For example, miR-486 is often referred to as "muscle-enriched" rather than "muscle-specific" because is expressed in other tissues as well [29]. In our study, we aimed to describe the miR signature that maternal low protein intake establishes in the skeletal muscle of male offspring. The skeletal muscle phenotypic differences between NN and NL groups was complemented with a profound change in $\mathrm{miR}$ signature, resulting in a clear distinguishable grouping of mice in PCA analysis and expression heatmaps. As expected, among the 92 DE miR that we found, after applying very strict statistical limits to ensure high accuracy of our results, myomiRs were included. Their expression levels during skeletal muscle development have been reported previously. miR-1 and miR-133a, for example, are upregulated by MyoD and MyoG during both human and mouse skeletal muscle differentiation in vitro $[76,77]$ and in vivo $[78,79]$ and also support myogenic differentiation [80,81]. Furthermore, miR-1/206/133 control embryonic myogenesis through regulation of BAF chromatin remodelling complexes [82]. Our findings are in agreement with these expression levels, i.e., miR-1 and -133a were significantly downregulated in the NL group compared with the $\mathrm{NN}$ group at weaning. It is also known that miR-1 and -206 suppress Pax7 expression and act as regulators of satellite cell proliferation and differentiation [81]. Postnatal muscle hypertrophy may also be regulated by satellite cell number per fibre at birth and their rate of proliferation as well as protein deposition (i.e., protein synthesis and degradation) [83]. Therefore, our future studies will focus on satellite cells which will be assessed in histological sections.

miR-206 was downregulated in NL mice and in LN mice too and validated by qPCR. It has been shown that miR-206 is highly expressed in newly formed muscle fibres, indicating that miR-206 may be involved in muscle regeneration and maturation [84]. Moreover, miR-206 directly targets Pax3, and inhibition with antagomirs results in delayed myogenic differentiation [85], but the miR-206 family is not essential for in vitro myogenesis, although it modulates the differentiation of skeletal myoblasts [86]. These results clearly demonstrate that the animal model used in the present study is valid and produces reliable sequencing data in accordance with the phenotypic outcome, since muscle size and $\mathrm{miR}$ expression are consistent with previous literature. Therefore, we proceeded to select and validate novel miRs that may have key functions in the nutrition-dependent developmental programming of skeletal muscle. It is reported that miR-15a has the highest expression level in skeletal muscle in comparison with other tissues [87] and is upregulated during human skeletal myoblast differentiation [88]. In addition, miR-34a targets thioredoxin reductase 2 (TXNRD2), which plays an important role in redox homeostasis of skeletal muscle [89] and is also involved in neuronal development [90]. Circulating miR-122 can enter muscle and adipose tissues in mice and reduce mRNA levels of genes involved in triglyceride synthesis and, thus, regulate energy balance [91]. Finally, miR-199a-3p expression is highly expressed in skeletal muscle and controls genes of the IGF1/Akt/mTOR signalling pathway to regulate $\mathrm{C} 2 \mathrm{C} 12$ myoblasts differentiation [92].

It has also been previously reported that foetal exposure to maternal diabetes is associated with increased skeletal muscle expression of miR-15a, and that this may contribute to development of metabolic disease in later life [93]. Furthermore, miR-15a expression is elevated in powerlifters as compared to normal human controls [94] showing a positive correlation with increased muscle mass and strength. miR-34a was also downregulated in NL mice and LN mice, as shown by qPCR. miR-34a has been shown to induce senescence of endothelial progenitor cells by inhibiting SIRT1, which results in increased levels of acetylated FOXO1 [95] and, thus, inhibits skeletal muscle development. Other studies suggest that Sirt1 antisense (AS) long non-coding RNA (lncRNA) interacts with its mRNA to inhibit muscle formation by attenuating function of miR-34a [96]. Additionally, several reports describe that miR-34a plays important roles in neuronal development [89]. 
Specifically, miR-34a has been shown to regulate spinal morphology and neurite outgrowth and is associated with both morphological and electrophysiological changes in mouse models [97]. Ectopic expression of miR-34a has also been shown to modulate neural differentiation by increasing the percentage of post-mitotic neurons and neurite elongation of mouse neural stem cells, whereas antagomirs of miR-34a had the opposite effect, suggesting that miR-34a is required for proper neuronal differentiation [98]. Differences in the timing of changes in muscle innervation during development between human and in small mammals have been reported, thus in human the transition from poly- to mononeuronal muscle innervation takes place between the 16 and 25 weeks of gestation, whereas in rodents these changes occur relatively later in the first two weeks after birth [99]. It is therefore possible that the reduction in miR-34a evident in both NL and LN groups is associated with dysregulation of muscle fibre innervation due to inadequate intake of protein during gestation or lactation and our future studies will focus on examining any specific changes occurring in muscle innervation in these mice phenotypically.

Although miR-122 is considered as a striated muscle-specific miR [25], its function has not been extensively explored. However, it is known to exhibit an inhibitory role of in the TGF $\beta /$ Smad signalling pathway in skeletal muscle fibrosis after contusion [100]. Finally, miR-199a regulates myogenic differentiation by acting downstream of Srf, which targets multiple factors within the Wnt signalling pathway [79]. Based on this literature, we selected miRs-15a, -34a, -122 and -199a and examined the DE of miR-seq by qPCR. Our bioinformatic approaches revealed that our validated miRs are predicted to target a variety of genes that regulate different developmental processes including striated muscle cell apoptosis and histone methylation, which is associated with epigenetic regulation of embryonic myogenesis [101]. Importantly, these miRs share a variety of common predicted gene targets that are implicated in skeletal muscle physiology with myomiRs. We, therefore, conclude that the four selected miRs can serve as a complement of known myomiRs and form a promising set to monitor skeletal muscle development, but further studies need to confirm this idea. Considering that tissue-specific miR expression can be affected by the nutritional status and, in this case, the maternal low protein consumption particularly during lactation, we can conclude that the DE of these miRs might be the cause of reduced myofibre size in NL mice. The present study also highlights the importance of a maternal balanced diet especially during lactation as this has a direct impact on offspring health. A limitation of our work is that we have not examined the role of our selected miRs in vitro, e.g., by using $\mathrm{C} 2 \mathrm{C} 12$ cells to evaluate fusion and myofibre formation, or in vivo by direct injections of miRs or antagomiRs and assessment of skeletal muscle integrity throughout life course. However, these approaches are beyond the scope of our present study and we intend to include them in our future work.

The increase in skeletal muscle mass after birth occurs primarily through muscle fibre hypertrophy given that fibre number is established during early postnatal development [102]. The amount of protein synthesised by a cell is dependent on translational capacity and efficiency. Ribosome biogenesis is as an important regulator of skeletal muscle growth and maintenance by changing the cellular translational capacity. There is a role for ribosome biogenesis in skeletal muscle growth during postnatal development [103]. In mammals, the ribosome is assembled from ribosomal RNAs (rRNAs), together with at least 80 different protein subunits [31]. Within the ribosome, 18S rRNA guides the decoding of the mRNA, whereas $28 \mathrm{~S}$ rRNA forms the core of the peptidyltransferase centre that polymerises the amino acid sequence encoded by the mRNA into functional proteins. Maturation post-transcriptionally of rRNAs is part of the biosynthesis of ribosomes [104]. 47S rRNA ribonucleolytic processing into mature $18 \mathrm{~S}, 5.8 \mathrm{~S}$ and $28 \mathrm{~S}$ rRNAs is rate-limiting for ribosome biogenesis [105]. We identified an increase in U3 in NL compared to NN. Whilst a network of snoRNAs is involved in key ribosome biogenesis processes [106], U3 is a factor in the generation of 18S rRNA [106]. This snoRNA is a highly abundant and evolutionarily conserved box C/D-class snoRNA, guiding the endoribonucleolytic processing of the $5^{\prime}$ external transcribed spacer (ETS) of the $47 \mathrm{~S}$ pre-rRNA by base complementarity-guided 
pre-rRNA substrate recognition [107]. It has a key role in the maturation of $18 \mathrm{~S}$ rRNA $[108,109]$. In cartilage, we demonstrated the global impact of reduced U3 expression on protein translational processes and inflammatory pathways and found that altering U3 expression has a direct effect on rRNA expression and translational capacity of chondrocytes. We showed that the extracellular environment is capable of controlling cellular U3 levels, thereby tuning the cell's capacity to generate mature rRNA species [110]. The increase in U3 in NL may be a compensatory method to protein restriction.

Although miRs have been extensively studied, very limited evidence refers to the role of non-miR sncRNAs in muscle development [111]. Several SNORDs, such as SNORD-116, -48, -84, -95 and -97, showed DE in skeletal muscle of Amyotrophic Lateral Sclerosis (ALS) patients as compared to healthy controls [112]. RNA-seq analysis using serum and muscle samples of Duchenne Muscular Dystrophy (DMD) patients also revealed altered snoRNA and snRNA levels, suggesting their use as potential biomarkers for DMD [113]. Here, we report that low protein intake during lactation in NL male mice results in DE of multiple snoRNAs of both C/D and H/ACA box subtypes with canonical roles, for the first time in the literature. Two snoRNAs (SNORD33 and SNORD35) were reduced in NL. Whilst these snoRNAs have canonical roles [114], they additionally have non-canonical roles as regulators of metabolic and oxidative stress pathways in mammalian cells as they were induced in vivo in response the generalised oxidative stress [115]. Loss of their host gene Rpl13a altered mitochondrial metabolism and lowered reactive oxygen species (ROS) production, but the snoRNAs are themselves regulated by reactive oxygen species and oxidative stress, with dynamic accumulation of snoRNAs in the cytoplasm in response to oxidative stress [116]. In human knee cartilage, we identified changes in these "oxi-snoRNAs" in ageing and osteoarthritis, and this was a cross species effect. Our in vitro experiments demonstrated that they respond to their environment and oxidative stress stimuli and using physiological levels of ROS generated using fibronectin fragments increased the expression these snoRNAs [117]. In skeletal muscle, suboptimal maternal nutrition followed by accelerated postnatal growth has been shown to induce an accelerated ageing phenotype and oxidative stress in adult offspring male rats. Specifically, Tarry-Adkins et al. demonstrated that rats born to dams fed on low protein chow but suckled postnatally by a dam maintained on a normal diet until weaning and maintained on a normal diet until 12 months of age have accelerated telomere shortening and increased DNA damage, which was associated with a strong oxidative stress phenotype, a compensatory increased antioxidant defence enzyme activity and inflammation [118]. The authors also demonstrated that mitochondrial dysfunction was evident in the muscle of the offspring by a reduction in citrate synthase activity (a marker of intact mitochondria) and increased Complex I, linked Complex II III and Complex IV electron transport chain (ETC) activity, suggesting that muscle mitochondria might have to compensate for fewer mitochondria by increasing the activity of ETC complexes to generate sufficient ATP, which in turn produces more ROS. Although we did not examine these changes directly in this study, we speculate that these ROS-related mechanistic changes occur as a consequence of suboptimal in utero and early environments and are affected directly by oxi-miRs and/or oxi-snoRNAs, and this in turn leads to the development of ROS induced age-related diseases including sarcopenia [118].

\section{Conclusions}

We here report that small RNA-seq reveals numerous sncRNAs which are correlated with reduced skeletal muscle size of $21 \mathrm{~d}$ male offspring that were lactated from mouse dams on low protein diet. We further describe four differentially expressed miRs, miRs$15 a,-34 a,-122$ and $-199 a$, that may be implicated in this process. These selected miRs could be combined with established myomiRs composing an interactive biomolecular network that may regulate this effect, as the bioinformatic approach revealed. Finally, to our knowledge, this is the first study exploring the role of snoRNAs in muscle development under restricted diet conditions. Although we do not provide a mechanistic explanation 
or a functional outcome of these alterations, our study suggests novel sncRNA signatures and identify potential candidates for innovative directions in muscle development. Future studies will facilitate uncovering the detailed molecular mechanisms.

Supplementary Materials: The following are available online at www.mdpi.com/20734409/10/5/1166/s1, Figure S1: Macroscopical differences between N and L pups, Figure S2: Predicted gene target analysis for combined selected miRs and myomiRs, Table S1: miR primer list used for qPCR validation of small RNA-seq data, Table S2: Differentially expressed miRs, Table S3: GO analysis using IPA, Table S4: Differentially expressed sncRNAs.

Author Contributions: I.K., A.V. and K.G.-W. designed the experiments with input from S.E.O. and M.J.P; I.K. and M.A. performed the experiments and acquired and analysed the data; L.F., I.M. and S.M. analysed the sequencing data; and I.K. drafted the manuscript, which was critically revised and approved by all co-authors. All authors have read and agreed to the published version of the manuscript.

Funding: This work was funded by the BBSRC (grant BB/P008429/1) to A.V. and K.G.-W. and departmental support from the Institute of Life Course and Medical Sciences, University of Liverpool to I.K. M.J.P. is funded through a Wellcome Trust Clinical Intermediate Fellowship (grant 107471/Z/15/Z). We thank the Biomedical Services Unit and the Centre for Genomic Research at the University of Liverpool. S.E.O. is a member of the MRC Metabolic Diseases Unit (MC_UU_00014/4).

Institutional Review Board Statement: All experimental protocols were performed according to the UK Animals (Scientific Procedures) Act 1986 regulations and obtained ethical approval from the University of Liverpool Animal Welfare Ethical Review Board (AWERB). All animal procedures were performed under a valid Home Office Project Licence.

Informed Consent Statement: Not applicable.

Data Availability Statement: Raw sequencing data for this study are stored at the Sequence Read Archive (SRA) using the BioProject accession: PRJNA668275.

Conflicts of Interest: The authors declare no conflict of interest.

\section{References}

1. Barker, D.J. The fetal and infant origins of disease. Eur. J. Clin. Invest. 1995, 25, 457-463, doi:10.1111/j.1365-2362.1995.tb01730.x.

2. Reed, S.A.; Raja, J.S.; Hoffman, M.L.; Zinn, S.A.; Govoni, K.E. Poor maternal nutrition inhibits muscle development in ovine offspring. J. Anim. Sci. Biotechnol. 2014, 5, 43, doi:10.1186/2049-1891-5-43.

3. Oreffo, R.O.; Lashbrooke, B.; Roach, H.I.; Clarke, N.M.; Cooper, C. Maternal protein deficiency affects mesenchymal stem cell activity in the developing offspring. Bone 2003, 33, 100-107, doi:10.1016/s8756-3282(03)00166-2.

4. Raja, J.S.; Hoffman, M.L.; Govoni, K.E.; Zinn, S.A.; Reed, S.A. Restricted maternal nutrition alters myogenic regulatory factor expression in satellite cells of ovine offspring. Animal 2016, 10, 1200-1203, doi:10.1017/S1751731116000070.

5. Du, M.; Zhao, J.X.; Yan, X.; Huang, Y.; Nicodemus, L.V.; Yue, W.; McCormick, R.J.; Zhu, M.J. Fetal muscle development, mesenchymal multipotent cell differentiation, and associated signaling pathways. J. Anim. Sci. 2011, 89, 583-590, doi:10.2527/jas.2010-3386.

6. Jousse, C.; Muranishi, Y.; Parry, L.; Montaurier, C.; Even, P.; Launay, J.M.; Carraro, V.; Maurin, A.C.; Averous, J.; Chaveroux, C.; et al. Perinatal protein malnutrition affects mitochondrial function in adult and results in a resistance to high fat diet-induced obesity. PLoS ONE 2014, 9, e104896, doi:10.1371/journal.pone.0104896.

7. Jansson, N.; Pettersson, J.; Haafiz, A.; Ericsson, A.; Palmberg, I.; Tranberg, M.; Ganapathy, V.; Powell, T.L.; Jansson, T. Downregulation of placental transport of amino acids precedes the development of intrauterine growth restriction in rats fed a low protein diet. J. Physiol. 2006, 576, 935-946, doi:10.1113/jphysiol.2006.116509.

8. Pantham, P.; Rosario, F.J.; Nijland, M.; Cheung, A.; Nathanielsz, P.W.; Powell, T.L.; Galan, H.L.; Li, C.; Jansson, T. Reduced placental amino acid transport in response to maternal nutrient restriction in the baboon. Am. J. Physiol. Regul. Integr. Comp. Physiol. 2015, 309, R740-R746, doi:10.1152/ajpregu.00161.2015.

9. Zhu, M.J.; Ford, S.P.; Nathanielsz, P.W.; Du, M. Effect of maternal nutrient restriction in sheep on the development of fetal skeletal muscle. Biol. Reprod. 2004, 71, 1968-1973, doi:10.1095/biolreprod.104.034561.

10. Zhu, M.J.; Ford, S.P.; Means, W.J.; Hess, B.W.; Nathanielsz, P.W.; Du, M. Maternal nutrient restriction affects properties of skeletal muscle in offspring. J. Physiol. 2006, 575, 241-250, doi:10.1113/jphysiol.2006.112110.

11. Confortim, H.D.; Jeronimo, L.C.; Centenaro, L.A.; Pinheiro, P.F.; Matheus, S.M.; Torrejais, M.M. Maternal protein restriction during pregnancy and lactation affects the development of muscle fibers and neuromuscular junctions in rats. Muscle Nerve 2017, 55, 109-115, doi:10.1002/mus.25187. 
12. Confortim, H.D.; Jeronimo, L.C.; Centenaro, L.A.; Felipe Pinheiro, P.F.; Brancalhao, R.M.; Michelin Matheus, S.M.; Torrejais, M.M. Effects of aging and maternal protein restriction on the muscle fibers morphology and neuromuscular junctions of rats after nutritional recovery. Micron 2015, 71, 7-13, doi:10.1016/j.micron.2014.12.006.

13. Beauchamp, B.; Ghosh, S.; Dysart, M.W.; Kanaan, G.N.; Chu, A.; Blais, A.; Rajamanickam, K.; Tsai, E.C.; Patti, M.E.; Harper, M.E. Low birth weight is associated with adiposity, impaired skeletal muscle energetics and weight loss resistance in mice. Int. J. Obes. (Lond.) 2015, 39, 702-711, doi:10.1038/ijo.2014.120.

14. Patel, H.P.; Jameson, K.A.; Syddall, H.E.; Martin, H.J.; Stewart, C.E.; Cooper, C.; Sayer, A.A. Developmental influences, muscle morphology, and sarcopenia in community-dwelling older men. J. Gerontol. A Biol. Sci. Med. Sci. 2012, 67, 82-87, doi:10.1093/gerona/glr020.

15. Godfrey, K.M.; Barker, D.J. Fetal nutrition and adult disease. Am J Clin Nutr 2000, 71, 1344S-1352S, doi:10.1093/ajcn/71.5.1344s.

16. Barker, D.J. The fetal origins of diseases of old age. Eur. J. Clin. Nutr. 1992, 46 (Suppl. 3), S3-S9.

17. Soriano-Arroquia, A.; House, L.; Tregilgas, L.; Canty-Laird, E.; Goljanek-Whysall, K. The functional consequences of age-related changes in microRNA expression in skeletal muscle. Biogerontology 2016, 17, 641-654, doi:10.1007/s10522-016-9638-8.

18. Goljanek-Whysall, K.; Sweetman, D.; Munsterberg, A.E. microRNAs in skeletal muscle differentiation and disease. Clin. Sci. (Lond.) 2012, 123, 611-625, doi:10.1042/CS20110634.

19. Goncalves, T.J.M.; Armand, A.S. Non-coding RNAs in skeletal muscle regeneration. Noncoding RNA Res. 2017, 2, 56-67, doi:10.1016/j.ncrna.2017.03.003.

20. Nie, M.; Deng, Z.L.; Liu, J.; Wang, D.Z. Noncoding RNAs, Emerging Regulators of Skeletal Muscle Development and Diseases. Biomed. Res. Int. 2015, 2015, 676575, doi:10.1155/2015/676575.

21. Goljanek-Whysall, K.; Pais, H.; Rathjen, T.; Sweetman, D.; Dalmay, T.; Munsterberg, A. Regulation of multiple target genes by miR-1 and miR-206 is pivotal for C2C12 myoblast differentiation. J. Cell Sci. 2012, 125, 3590-3600, doi:10.1242/jcs.101758.

22. Brown, D.M.; Goljanek-Whysall, K. microRNAs: Modulators of the underlying pathophysiology of sarcopenia? Ageing Res. Rev. 2015, 24, 263-273, doi:10.1016/j.arr.2015.08.007.

23. McGregor, R.A.; Poppitt, S.D.; Cameron-Smith, D. Role of microRNAs in the age-related changes in skeletal muscle and diet or exercise interventions to promote healthy aging in humans. Ageing Res. Rev. 2014, 17, 25-33, doi:10.1016/j.arr.2014.05.001.

24. Lee, R.C.; Ambros, V. An extensive class of small RNAs in Caenorhabditis elegans. Science 2001, 294, 862-864, doi:10.1126/science.1065329.

25. Lagos-Quintana, M.; Rauhut, R.; Yalcin, A.; Meyer, J.; Lendeckel, W.; Tuschl, T. Identification of tissue-specific microRNAs from mouse. Curr. Biol. 2002, 12, 735-739, doi:10.1016/s0960-9822(02)00809-6.

26. McCarthy, J.J. MicroRNA-206: The skeletal muscle-specific myomiR. Biochim. Biophys. Acta 2008, 1779, 682-691, doi:10.1016/j.bbagrm.2008.03.001.

27. Sempere, L.F.; Freemantle, S.; Pitha-Rowe, I.; Moss, E.; Dmitrovsky, E.; Ambros, V. Expression profiling of mammalian microRNAs uncovers a subset of brain-expressed microRNAs with possible roles in murine and human neuronal differentiation. Genome Biol. 2004, 5, R13, doi:10.1186/gb-2004-5-3-r13.

28. van Rooij, E.; Quiat, D.; Johnson, B.A.; Sutherland, L.B.; Qi, X.; Richardson, J.A.; Kelm, R.J., Jr.; Olson, E.N. A family of microRNAs encoded by myosin genes governs myosin expression and muscle performance. Dev. Cell 2009, 17, 662-673, doi:10.1016/j.devcel.2009.10.013.

29. Small, E.M.; O’Rourke, J.R.; Moresi, V.; Sutherland, L.B.; McAnally, J.; Gerard, R.D.; Richardson, J.A.; Olson, E.N. Regulation of PI3-kinase/Akt signaling by muscle-enriched microRNA-486. Proc. Natl. Acad. Sci. USA 2010, 107, 4218-4223, doi:10.1073/pnas.1000300107.

30. Horak, M.; Novak, J.; Bienertova-Vasku, J. Muscle-specific microRNAs in skeletal muscle development. Dev. Biol. 2016, 410, 113, doi:10.1016/j.ydbio.2015.12.013.

31. Khatter, H.; Myasnikov, A.G.; Natchiar, S.K.; Klaholz, B.P. Structure of the human 80 S ribosome. Nature 2015, 520, 640-645, doi:10.1038/nature14427.

32. Jady, B.E.; Kiss, T. A small nucleolar guide RNA functions both in 2'-O-ribose methylation and pseudouridylation of the U5 spliceosomal RNA. EMBO J. 2001, 20, 541-551, doi:10.1093/emboj/20.3.541.

33. Cavaille, J.; Nicoloso, M.; Bachellerie, J.P. Targeted ribose methylation of RNA in vivo directed by tailored antisense RNA guides. Nature 1996, 383, 732-735, doi:10.1038/383732a0.

34. Ganot, P.; Bortolin, M.L.; Kiss, T. Site-specific pseudouridine formation in preribosomal RNA is guided by small nucleolar RNAs. Cell 1997, 89, 799-809, doi:10.1016/s0092-8674(00)80263-9.

35. Peffers, M.J.; Chabronova, A.; Balaskas, P.; Fang, Y.; Dyer, P.; Cremers, A.; Emans, P.J.; Feczko, P.Z.; Caron, M.M.; Welting, T.J.M. SnoRNA signatures in cartilage ageing and osteoarthritis. Sci. Rep. 2020, 10, 10641, doi:10.1038/s41598-020-67446-z.

36. Stepanov, G.A.; Filippova, J.A.; Komissarov, A.B.; Kuligina, E.V.; Richter, V.A.; Semenov, D.V. Regulatory role of small nucleolar RNAs in human diseases. Biomed. Res. Int. 2015, 2015, 206849, doi:10.1155/2015/206849.

37. Falaleeva, M.; Welden, J.R.; Duncan, M.J.; Stamm, S. C/D-box snoRNAs form methylating and non-methylating ribonucleoprotein complexes: Old dogs show new tricks. Bioessays 2017, 39, doi:10.1002/bies.201600264.

38. Maniatis, T.; Reed, R. The role of small nuclear ribonucleoprotein particles in pre-mRNA splicing. Nature 1987, 325, 673-678, doi:10.1038/325673a0.

39. Buratti, E.; Baralle, F.E. The multiple roles of TDP-43 in pre-mRNA processing and gene expression regulation. RNA Biol. 2010, 7, 420-429, doi:10.4161/rna.7.4.12205. 
40. Ideue, T.; Adachi, S.; Naganuma, T.; Tanigawa, A.; Natsume, T.; Hirose, T. U7 small nuclear ribonucleoprotein represses histone gene transcription in cell cycle-arrested cells. Proc. Natl. Acad. Sci. USA 2012, 109, 5693-5698, doi:10.1073/pnas.1200523109.

41. Lasda, E.L.; Blumenthal, T. Trans-splicing. Wiley Interdiscip. Rev. RNA 2011, 2, 417-434, doi:10.1002/wrna.71.

42. Kozomara, A.; Birgaoanu, M.; Griffiths-Jones, S. miRBase: From microRNA sequences to function. Nucleic Acids Res. 2019, 47, D155-D162, doi:10.1093/nar/gky1141.

43. Fowler, E.K.; Bradley, T.; Moxon, S.; Chapman, T. Divergence in transcriptional and regulatory responses to mating in male and female fruitflies. Sci. Rep. 2019, 9, 16100, doi.org/10.1038/s41598-019-51141-9.

44. Prufer, K.; Stenzel, U.; Dannemann, M.; Green, R.E.; Lachmann, M.; Kelso, J. PatMaN: Rapid alignment of short sequences to large databases. Bioinformatics 2008, 24, 1530-1531, doi:10.1093/bioinformatics/btn223.

45. Love, M.I.; Huber, W.; Anders, S. Moderated estimation of fold change and dispersion for RNA-seq data with DESeq2. Genome Biol. 2014, 15, 550, doi:10.1186/s13059-014-0550-8.

46. Ge, S.X.; Son, E.W.; Yao, R. iDEP: An integrated web application for differential expression and pathway analysis of RNA-Seq data. BMC Bioinform. 2018, 19, 534, doi:10.1186/s12859-018-2486-6.

47. Yates, A.D.; Achuthan, P.; Akanni, W.; Allen, J.; Allen, J.; Alvarez-Jarreta, J.; Amode, M.R.; Armean, I.M.; Azov, A.G.; Bennett, R.; et al. Ensembl 2020. Nucleic Acids Res. 2020, 48, D682-D688, doi:10.1093/nar/gkz966.

48. Quinlan, A.R.; Hall, I.M. BEDTools: A flexible suite of utilities for comparing genomic features. Bioinformatics 2010, 26, 841-842, doi:10.1093/bioinformatics/btq033.

49. Metsalu, T.; Vilo, J. ClustVis: A web tool for visualizing clustering of multivariate data using Principal Component Analysis and heatmap. Nucleic Acids Res. 2015, 43, W566-W570, doi:10.1093/nar/gkv468.

50. Benjamini, Y.; Drai, D.; Elmer, G.; Kafkafi, N.; Golani, I. Controlling the false discovery rate in behavior genetics research. Behav. Brain Res. 2001, 125, 279-284, doi:10.1016/s0166-4328(01)00297-2.

51. Proctor, C.J.; Goljanek-Whysall, K. Using computer simulation models to investigate the most promising microRNAs to improve muscle regeneration during ageing. Sci. Rep. 2017, 7, 12314, doi:10.1038/s41598-017-12538-6.

52. Peffers, M.J.; Goljanek-Whysall, K.; Collins, J.; Fang, Y.; Rushton, M.; Loughlin, J.; Proctor, C.; Clegg, P.D. Decoding the Regulatory Landscape of Ageing in Musculoskeletal Engineered Tissues Using Genome-Wide DNA Methylation and RNASeq. PLoS ONE 2016, 11, e0160517, doi:10.1371/journal.pone.0160517.

53. Livak, K.J.; Schmittgen, T.D. Analysis of relative gene expression data using real-time quantitative PCR and the 2(-Delta Delta C(T)) Method. Methods 2001, 25, 402-408, doi:10.1006/meth.2001.1262.

54. Agarwal, V.; Bell, G.W.; Nam, J.W.; Bartel, D.P. Predicting effective microRNA target sites in mammalian mRNAs. eLife 2015, 4, doi:10.7554/eLife.05005.

55. Dweep, H.; Gretz, N. miRWalk2.0: A comprehensive atlas of microRNA-target interactions. Nat. Methods 2015, 12, 697, doi:10.1038/nmeth.3485.

56. Wong, N.; Wang, X. miRDB: An online resource for microRNA target prediction and functional annotations. Nucleic Acids Res. 2015, 43, D146-D152, doi:10.1093/nar/gku1104.

57. Hsu, S.D.; Lin, F.M.; Wu, W.Y.; Liang, C.; Huang, W.C.; Chan, W.L.; Tsai, W.T.; Chen, G.Z.; Lee, C.J.; Chiu, C.M.; et al. miRTarBase: A database curates experimentally validated microRNA-target interactions. Nucleic Acids Res. 2011, 39, D163-D169, doi:10.1093/nar/gkq1107.

58. Shannon, P.; Markiel, A.; Ozier, O.; Baliga, N.S.; Wang, J.T.; Ramage, D.; Amin, N.; Schwikowski, B.; Ideker, T. Cytoscape: A software environment for integrated models of biomolecular interaction networks. Genome Res. 2003, 13, 2498-2504, doi:10.1101/gr.1239303.

59. Bindea, G.; Mlecnik, B.; Hackl, H.; Charoentong, P.; Tosolini, M.; Kirilovsky, A.; Fridman, W.H.; Pages, F.; Trajanoski, Z.; Galon, J. ClueGO: A Cytoscape plug-in to decipher functionally grouped gene ontology and pathway annotation networks. Bioinformatics 2009, 25, 1091-1093, doi:10.1093/bioinformatics/btp101.

60. Kanehisa, M.; Goto, S. KEGG: Kyoto encyclopedia of genes and genomes. Nucleic Acids Res. 2000, 28, 27-30, doi:10.1093/nar/28.1.27.

61. Vickers, M.H.; Ikenasio, B.A.; Breier, B.H. IGF-I treatment reduces hyperphagia, obesity, and hypertension in metabolic disorders induced by fetal programming. Endocrinology 2001, 142, 3964-3973, doi:10.1210/endo.142.9.8390.

62. Fahey, A.J.; Brameld, J.M.; Parr, T.; Buttery, P.J. The effect of maternal undernutrition before muscle differentiation on the muscle fiber development of the newborn lamb. J. Anim. Sci. 2005, 83, 2564-2571, doi:10.2527/2005.83112564x.

63. Mallinson, J.E.; Sculley, D.V.; Craigon, J.; Plant, R.; Langley-Evans, S.C.; Brameld, J.M. Fetal exposure to a maternal low-protein diet during mid-gestation results in muscle-specific effects on fibre type composition in young rats. Br. J. Nutr. 2007, 98, 292299, doi:10.1017/S0007114507701678.

64. Toscano, A.E.; Manhaes-de-Castro, R.; Canon, F. Effect of a low-protein diet during pregnancy on skeletal muscle mechanical properties of offspring rats. Nutrition 2008, 24, 270-278, doi:10.1016/j.nut.2007.12.004.

65. Bayol, S.; Jones, D.; Goldspink, G.; Stickland, N.C. The influence of undernutrition during gestation on skeletal muscle cellularity and on the expression of genes that control muscle growth. Br. J. Nutr. 2004, 91, 331-339, doi:10.1079/BJN20031070.

66. Ozanne, S.E.; Olsen, G.S.; Hansen, L.L.; Tingey, K.J.; Nave, B.T.; Wang, C.L.; Hartil, K.; Petry, C.J.; Buckley, A.J.; MosthafSeedorf, L. Early growth restriction leads to down regulation of protein kinase $\mathrm{C}$ zeta and insulin resistance in skeletal muscle. J. Endocrinol. 2003, 177, 235-241, doi:10.1677/joe.0.1770235. 
67. Chen, J.H.; Martin-Gronert, M.S.; Tarry-Adkins, J.; Ozanne, S.E. Maternal protein restriction affects postnatal growth and the expression of key proteins involved in lifespan regulation in mice. PLoS ONE 2009, 4, e4950, doi:10.1371/journal.pone.0004950.

68. Moss, F.P.; Leblond, C.P. Satellite cells as the source of nuclei in muscles of growing rats. Anat. Rec. 1971, 170, 421-435, doi:10.1002/ar.1091700405.

69. Miyazaki, M.; Esser, K.A. Cellular mechanisms regulating protein synthesis and skeletal muscle hypertrophy in animals. J. Appl. Physiol. (1985) 2009, 106, 1367-1373, doi:10.1152/japplphysiol.91355.2008.

70. Rehfeldt, C.; Nissen, P.M.; Kuhn, G.; Vestergaard, M.; Ender, K.; Oksbjerg, N. Effects of maternal nutrition and porcine growth hormone $(\mathrm{pGH})$ treatment during gestation on endocrine and metabolic factors in sows, fetuses and pigs, skeletal muscle development, and postnatal growth. Domest. Anim. Endocrinol. 2004, 27, 267-285, doi:10.1016/j.domaniend.2004.06.005.

71. Rayne, J.; Crawford, G.N. Increase in fibre numbers of the rat pterygoid muscles during postnatal growth. J. Anat. 1975, 119, 347-357.

72. Summers, P.J.; Medrano, J.F. Delayed myogenesis associated with muscle fiber hyperplasia in high-growth mice. Proc. Soc. Exp. Biol. Med. 1997, 214, 380-385, doi:10.3181/00379727-214-44107.

73. Pileggi, C.A.; Hedges, C.P.; Segovia, S.A.; Markworth, J.F.; Durainayagam, B.R.; Gray, C.; Zhang, X.D.; Barnett, M.P.; Vickers, M.H.; Hickey, A.J.; et al. Maternal High Fat Diet Alters Skeletal Muscle Mitochondrial Catalytic Activity in Adult Male Rat Offspring. Front. Physiol. 2016, 7, 546, doi:10.3389/fphys.2016.00546.

74. Bayol, S.A.; Simbi, B.H.; Stickland, N.C. A maternal cafeteria diet during gestation and lactation promotes adiposity and impairs skeletal muscle development and metabolism in rat offspring at weaning. J. Physiol. 2005, 567, 951-961, doi:10.1113/jphysiol.2005.088989.

75. Alfaradhi, M.Z.; Ozanne, S.E. Developmental programming in response to maternal overnutrition. Front. Genet. 2011, 2, 27, doi:10.3389/fgene.2011.00027.

76. Koutsoulidou, A.; Mastroyiannopoulos, N.P.; Furling, D.; Uney, J.B.; Phylactou, L.A. Expression of miR-1, miR-133a, miR-133b and miR-206 increases during development of human skeletal muscle. BMC Dev. Biol. 2011, 11, 34, doi:10.1186/1471-213X-11-34.

77. Rao, P.K.; Kumar, R.M.; Farkhondeh, M.; Baskerville, S.; Lodish, H.F. Myogenic factors that regulate expression of musclespecific microRNAs. Proc. Natl. Acad. Sci. USA 2006, 103, 8721-8726, doi:10.1073/pnas.0602831103.

78. Butchart, L.C.; Fox, A.; Shavlakadze, T.; Grounds, M.D. The long and short of non-coding RNAs during post-natal growth and differentiation of skeletal muscles: Focus on lncRNA and miRNAs. Differentiation 2016, 92, 237-248, doi:10.1016/j.diff.2016.05.003.

79. Lamon, S.; Zacharewicz, E.; Butchart, L.C.; Orellana, L.; Mikovic, J.; Grounds, M.D.; Russell, A.P. MicroRNA expression patterns in post-natal mouse skeletal muscle development. BMC Genomics 2017, 18, 52, doi:10.1186/s12864-016-3399-2.

80. Chen, J.F.; Mandel, E.M.; Thomson, J.M.; Wu, Q.; Callis, T.E.; Hammond, S.M.; Conlon, F.L.; Wang, D.Z. The role of microRNA1 and microRNA-133 in skeletal muscle proliferation and differentiation. Nat. Genet. 2006, 38, 228-233, doi:10.1038/ng1725.

81. Chen, J.F.; Tao, Y.; Li, J.; Deng, Z.; Yan, Z.; Xiao, X.; Wang, D.Z. microRNA-1 and microRNA-206 regulate skeletal muscle satellite cell proliferation and differentiation by repressing Pax7. J. Cell. Biol. 2010, 190, 867-879, doi:10.1083/jcb.200911036.

82. Goljanek-Whysall, K.; Mok, G.F.; Fahad Alrefaei, A.; Kennerley, N.; Wheeler, G.N.; Munsterberg, A. myomiR-dependent switching of BAF60 variant incorporation into Brg1 chromatin remodeling complexes during embryo myogenesis. Development 2014, 141, 3378-3387, doi:10.1242/dev.108787.

83. Gattazzo, F.; Laurent, B.; Relaix, F.; Rouard, H.; Didier, N. Distinct Phases of Postnatal Skeletal Muscle Growth Govern the Progressive Establishment of Muscle Stem Cell Quiescence. Stem Cell Rep. 2020, 15, 597-611, doi:10.1016/j.stemcr.2020.07.011.

84. Yuasa, K.; Hagiwara, Y.; Ando, M.; Nakamura, A.; Takeda, S.; Hijikata, T. MicroRNA-206 is highly expressed in newly formed muscle fibers: Implications regarding potential for muscle regeneration and maturation in muscular dystrophy. Cell Struct. Funct. 2008, 33, 163-169, doi:10.1247/csf.08022.

85. Goljanek-Whysall, K.; Sweetman, D.; Abu-Elmagd, M.; Chapnik, E.; Dalmay, T.; Hornstein, E.; Munsterberg, A. MicroRNA regulation of the paired-box transcription factor Pax3 confers robustness to developmental timing of myogenesis. Proc. Natl. Acad. Sci. USA 2011, 108, 11936-11941, doi:10.1073/pnas.1105362108.

86. Przanowska, R.K.; Sobierajska, E.; Su, Z.; Jensen, K.; Przanowski, P.; Nagdas, S.; Kashatus, J.A.; Kashatus, D.F.; Bhatnagar, S.; Lukens, J.R.; et al. miR-206 family is important for mitochondrial and muscle function, but not essential for myogenesis in vitro. FASEB J. 2020, 34, 7687-7702, doi:10.1096/fj.201902855RR.

87. Besnier, M.; Shantikumar, S.; Anwar, M.; Dixit, P.; Chamorro-Jorganes, A.; Sweaad, W.; Sala-Newby, G.; Madeddu, P.; Thomas, A.C.; Howard, L.; et al. miR-15a/-16 Inhibit Angiogenesis by Targeting the Tie2 Coding Sequence: Therapeutic Potential of a miR-15a/16 Decoy System in Limb Ischemia. Mol. Ther. Nucleic Acids 2019, 17, 49-62, doi:10.1016/j.omtn.2019.05.002.

88. Meyer, S.U.; Thirion, C.; Polesskaya, A.; Bauersachs, S.; Kaiser, S.; Krause, S.; Pfaffl, M.W. TNF-alpha and IGF1 modify the microRNA signature in skeletal muscle cell differentiation. Cell Commun. Signal. 2015, 13, 4, doi:10.1186/s12964-015-0083-0.

89. Smith-Vikos, T.; Slack, F.J. MicroRNAs and their roles in aging. J. Cell Sci 2012, 125, 7-17, doi:10.1242/jcs.099200.

90. Rokavec, M.; Li, H.; Jiang, L.; Hermeking, H. The p53/miR-34 axis in development and disease. J. Mol. Cell Biol. 2014, 6, 214-230, doi:10.1093/jmcb/mju003.

91. Chai, C.; Rivkin, M.; Berkovits, L.; Simerzin, A.; Zorde-Khvalevsky, E.; Rosenberg, N.; Klein, S.; Yaish, D.; Durst, R.; Shpitzen, S.; et al. Metabolic Circuit Involving Free Fatty Acids, microRNA 122, and Triglyceride Synthesis in Liver and Muscle Tissues. Gastroenterology 2017, 153, 1404-1415, doi:10.1053/j.gastro.2017.08.013. 
92. Jia, L.; Li, Y.F.; Wu, G.F.; Song, Z.Y.; Lu, H.Z.; Song, C.C.; Zhang, Q.L.; Zhu, J.Y.; Yang, G.S.; Shi, X.E. MiRNA-199a-3p regulates C2C12 myoblast differentiation through IGF-1/AKT/mTOR signal pathway. Int. J. Mol. Sci. 2013, 15, 296-308, doi:10.3390/ijms15010296.

93. Houshmand-Oeregaard, A.; Schrolkamp, M.; Kelstrup, L.; Hansen, N.S.; Hjort, L.; Thuesen, A.C.B.; Broholm, C.; Mathiesen, E.R.; Clausen, T.D.; Vaag, A.; et al. Increased expression of microRNA-15a and microRNA-15b in skeletal muscle from adult offspring of women with diabetes in pregnancy. Hum. Mol. Genet. 2018, 27, 1763-1771, doi:10.1093/hmg/ddy085.

94. D’Souza, R.F.; Bjornsen, T.; Zeng, N.; Aasen, K.M.M.; Raastad, T.; Cameron-Smith, D.; Mitchell, C.J. MicroRNAs in Muscle: Characterizing the Powerlifter Phenotype. Front. Physiol. 2017, 8, 383, doi:10.3389/fphys.2017.00383.

95. Zhao, T.; Li, J.; Chen, A.F. MicroRNA-34a induces endothelial progenitor cell senescence and impedes its angiogenesis via suppressing silent information regulator 1. Am. J. Physiol. Endocrinol. Metab. 2010, 299, E110-E116, doi:10.1152/ajpendo.00192.2010.

96. Wang, G.Q.; Wang, Y.; Xiong, Y.; Chen, X.C.; Ma, M.L.; Cai, R.; Gao, Y.; Sun, Y.M.; Yang, G.S.; Pang, W.J. Sirt1 AS lncRNA interacts with its mRNA to inhibit muscle formation by attenuating function of miR-34a. Sci. Rep. 2016, 6, 21865, doi:10.1038/srep21865.

97. Agostini, M.; Tucci, P.; Steinert, J.R.; Shalom-Feuerstein, R.; Rouleau, M.; Aberdam, D.; Forsythe, I.D.; Young, K.W.; Ventura, A.; Concepcion, C.P.; et al. microRNA-34a regulates neurite outgrowth, spinal morphology, and function. Proc. Natl. Acad. Sci. USA 2011, 108, 21099-21104, doi:10.1073/pnas.1112063108.

98. Aranha, M.M.; Santos, D.M.; Sola, S.; Steer, C.J.; Rodrigues, C.M. miR-34a regulates mouse neural stem cell differentiation. PLoS ONE 2011, 6, e21396, doi:10.1371/journal.pone.0021396.

99. Hesselmans, L.F.; Jennekens, F.G.; Van den Oord, C.J.; Veldman, H.; Vincent, A. Development of innervation of skeletal muscle fibers in man: Relation to acetylcholine receptors. Anat. Rec. 1993, 236, 553-562, doi:10.1002/ar.1092360315.

100. Sun, Y.; Wang, H.; Li, Y.; Liu, S.; Chen, J.; Ying, H. miR-24 and miR-122 Negatively Regulate the Transforming Growth Factorbeta/Smad Signaling Pathway in Skeletal Muscle Fibrosis. Mol. Ther. Nucleic Acids 2018, 11, 528-537, doi:10.1016/j.omtn.2018.04.005.

101. Jin, W.; Peng, J.; Jiang, S. The epigenetic regulation of embryonic myogenesis and adult muscle regeneration by histone methylation modification. Biochem. Biophys. Rep. 2016, 6, 209-219, doi:10.1016/j.bbrep.2016.04.009.

102. Lompre, A.M.; Schwartz, K.; d'Albis, A.; Lacombe, G.; Van Thiem, N.; Swynghedauw, B. Myosin isoenzyme redistribution in chronic heart overload. Nature 1979, 282, 105-107, doi:10.1038/282105a0.

103. Figueiredo, V.C.; McCarthy, J.J. Regulation of Ribosome Biogenesis in Skeletal Muscle Hypertrophy. Physiology (Bethesda) 2019, 34, 30-42, doi:10.1152/physiol.00034.2018.

104. Mullineux, S.T.; Lafontaine, D.L. Mapping the cleavage sites on mammalian pre-rRNAs: Where do we stand? Biochimie 2012, 94, 1521-1532, doi:10.1016/j.biochi.2012.02.001.

105. Henras, A.K.; Plisson-Chastang, C.; O’Donohue, M.F.; Chakraborty, A.; Gleizes, P.E. An overview of pre-ribosomal RNA processing in eukaryotes. Wiley Interdiscip. Rev. RNA 2015, 6, 225-242, doi:10.1002/wrna.1269.

106. Lafontaine, D.L. Noncoding RNAs in eukaryotic ribosome biogenesis and function. Nat. Struct. Mol. Biol. 2015, 22, 11-19, doi:10.1038/nsmb.2939.

107. Beltrame, M.; Tollervey, D. Base pairing between U3 and the pre-ribosomal RNA is required for $18 \mathrm{~S}$ rRNA synthesis. EMBO J. 1995, 14, 4350-4356.

108. Langhendries, J.L.; Nicolas, E.; Doumont, G.; Goldman, S.; Lafontaine, D.L. The human box C/D snoRNAs U3 and U8 are required for pre-rRNA processing and tumorigenesis. Oncotarget 2016, 7, 59519-59534, doi:10.18632/oncotarget.11148.

109. Kass, S.; Tyc, K.; Steitz, J.A.; Sollner-Webb, B. The U3 small nucleolar ribonucleoprotein functions in the first step of preribosomal RNA processing. Cell 1990, 60, 897-908, doi:10.1016/0092-8674(90)90338-f.

110. Ripmeester, E.G.J.; Caron, M.M.J.; van den Akker, G.G.H.; Surtel, D.A.M.; Cremers, A.; Balaskas, P.; Dyer, P.; Housmans, B.A.C.; Chabronova, A.; Smagul, A.; et al. Impaired chondrocyte U3 snoRNA expression in osteoarthritis impacts the chondrocyte protein translation apparatus. Sci. Rep. 2020, 10, 13426, doi:10.1038/s41598-020-70453-9.

111. Ballarino, M.; Morlando, M.; Fatica, A.; Bozzoni, I. Non-coding RNAs in muscle differentiation and musculoskeletal disease. J. Clin. Invest. 2016, 126, 2021-2030, doi:10.1172/JCI84419.

112. Kovanda, A.; Leonardis, L.; Zidar, J.; Koritnik, B.; Dolenc-Groselj, L.; Ristic Kovacic, S.; Curk, T.; Rogelj, B. Differential expression of microRNAs and other small RNAs in muscle tissue of patients with ALS and healthy age-matched controls. Sci. Rep. 2018, 8, 5609, doi:10.1038/s41598-018-23139-2.

113. Coenen-Stass, A.M.L.; Sork, H.; Gatto, S.; Godfrey, C.; Bhomra, A.; Krjutskov, K.; Hart, J.R.; Westholm, J.O.; O’Donovan, L.; Roos, A.; et al. Comprehensive RNA-Sequencing Analysis in Serum and Muscle Reveals Novel Small RNA Signatures with Biomarker Potential for DMD. Mol. Ther. Nucleic Acids 2018, 13, 1-15, doi:10.1016/j.omtn.2018.08.005.

114. Lestrade, L.; Weber, M.J. snoRNA-LBME-db, a comprehensive database of human H/ACA and C/D box snoRNAs. Nucleic Acids Res. 2006, 34, D158-D162, doi:10.1093/nar/gkj002.

115. Michel, C.I.; Holley, C.L.; Scruggs, B.S.; Sidhu, R.; Brookheart, R.T.; Listenberger, L.L.; Behlke, M.A.; Ory, D.S.; Schaffer, J.E. Small nucleolar RNAs U32a, U33, and U35a are critical mediators of metabolic stress. Cell Metab. 2011, 14, 33-44, doi:10.1016/j.cmet.2011.04.009.

116. Holley, C.L.; Li, M.W.; Scruggs, B.S.; Matkovich, S.J.; Ory, D.S.; Schaffer, J.E. Cytosolic accumulation of small nucleolar RNAs (snoRNAs) is dynamically regulated by NADPH oxidase. J. Biol. Chem. 2015, 290, 11741-11748, doi:10.1074/jbc.M115.637413. 
117. Pimlott, Z.; Hontoir, F.; Ashraf Kharaz, Y.; Anderson, J.; Dyer, P.; Collins, J.; Loeser, R.; Welting, T.; Caron, M.; Peffers, M.J. Small nucleolar RNAs as mediators of oxidative stress in cross species cartilage and osteoarthritis. Osteoarthr. Cartil. 2020, 28, S342, doi:10.1016/j.joca.2020.02.532.

118. Tarry-Adkins, J.L.; Fernandez-Twinn, D.S.; Chen, J.H.; Hargreaves, I.P.; Neergheen, V.; Aiken, C.E.; Ozanne, S.E. Poor maternal nutrition and accelerated postnatal growth induces an accelerated aging phenotype and oxidative stress in skeletal muscle of male rats. Dis. Model. Mech. 2016, 9, 1221-1229, doi:10.1242/dmm.026591. 